This item was submitted to Loughborough's Research Repository by the author.

Items in Figshare are protected by copyright, with all rights reserved, unless otherwise indicated.

\title{
Angular (pitch and yaw) vibration measurements directly from rotors using laser vibrometry
}

PLEASE CITE THE PUBLISHED VERSION

http://dx.doi.org/10.1016/j.ymssp.2014.01.013

PUBLISHER

(C) Elsevier Ltd.

VERSION

AM (Accepted Manuscript)

LICENCE

CC BY-NC-ND 4.0

REPOSITORY RECORD

Halkon, Ben, and Steve Rothberg. 2014. "Angular (pitch and Yaw) Vibration Measurements Directly from Rotors Using Laser Vibrometry”. Loughborough University. https://hdl.handle.net/2134/14186. 
This item was submitted to Loughborough's Institutional Repository (https://dspace.lboro.ac.uk/) by the author and is made available under the following Creative Commons Licence conditions.

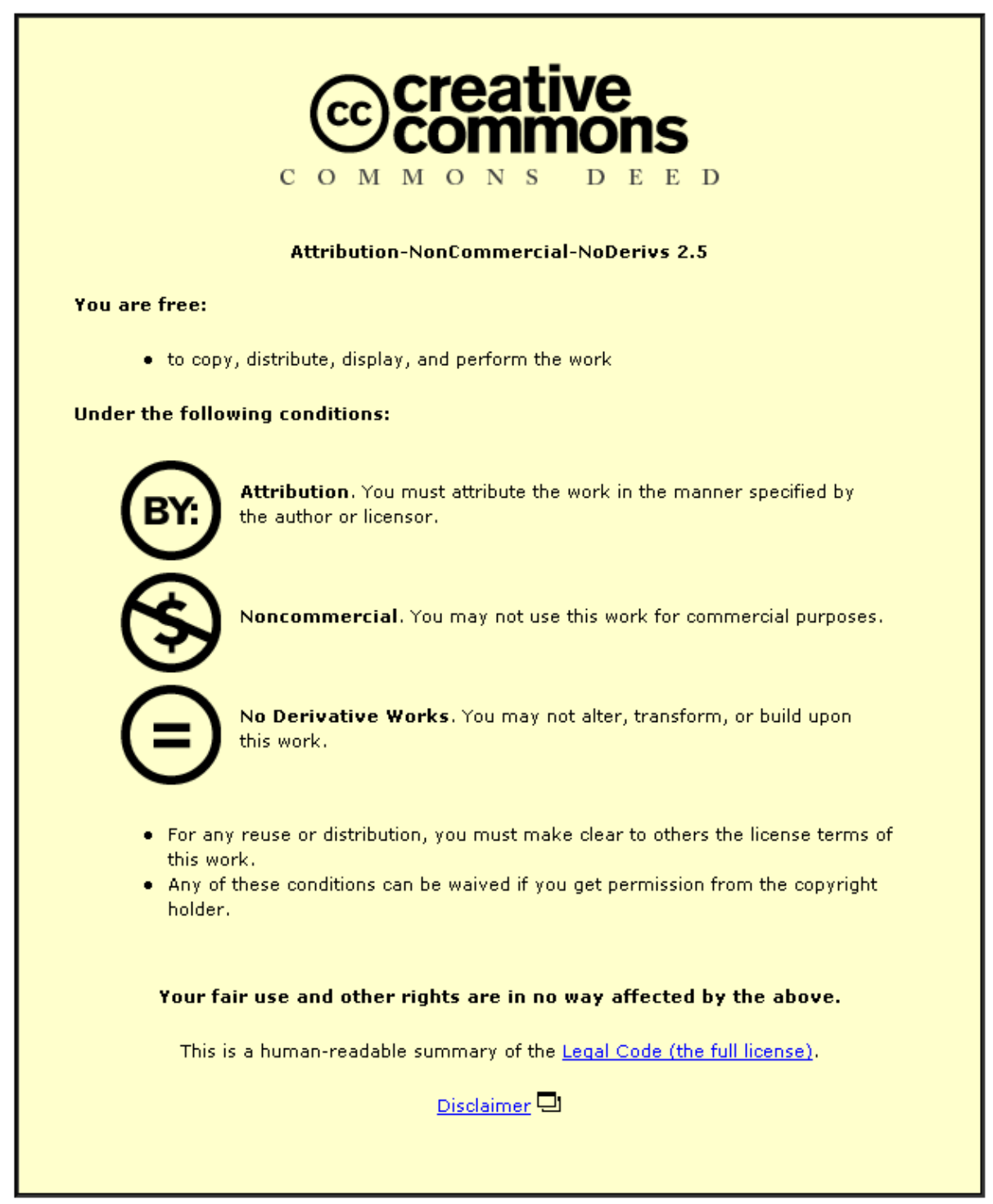

For the full text of this licence, please go to: http://creativecommons.org/licenses/by-nc-nd/2.5/ 


\section{Angular (pitch and yaw) vibration measurements directly from rotors using laser vibrometry}

Ben J. Halkon, Steve J. Rothberg

Wolfson School of Mechanical and Manufacturing Engineering

Loughborough University, Loughborough, Leicestershire, LE11 3TU, UK.

Corresponding author: Ben Halkon

e-mail: b.j.halkon@lboro.ac.uk,

tel: +44 (0) 1509564823 


\title{
Angular (pitch and yaw) vibration measurements directly from rotors using laser vibrometry
}

\author{
Ben J. Halkon, Steve J. Rothberg \\ Wolfson School of Mechanical and Manufacturing Engineering \\ Loughborough University, UK
}

\begin{abstract}
Parallel beam laser vibrometers offer direct measurement of pitch and yaw vibration directly from rotors. This paper, intended as an essential guide for the practical parallel beam laser vibrometer practioner, presents exact mathematical expressions for measured angular velocity in the presence of inevitable misalignments and estimates the likely measurement error levels due to such misalignments as well as to other sources of uncertainty through numerical simulation. Cross-sensitivity to the orthogonal vibration component, i.e. cross-sensitivity in a pitch measurement to yaw motion and viceversa, is confirmed for rough rotors whereas it is shown not to be present when rotors are polishedcircular. A complementary experimental investigation of the relationship between surface roughness and cross-sensitivity confirms the identification of two preferred measurement configurations: from the side of a polished-circular rotor and from the end face of a (rough) rotor coated in retro-reflective tape. Rotors with surface roughness up to $50 \mathrm{~nm}$ satisfy the former case provided the vibration displacement at the rotor surface does not exceed $20 \%$ beam diameter. For surfaces with roughness of $10 \mathrm{~nm}$ this can be extended to $50 \%$. For rough rotor end face measurements, post-processing is required to resolve the inherent cross-sensitivity; the need for post-processing is justified quantitatively through numerical simulation. Further simulations incorporating typical levels of instrument misalignment and measurement noise are used to enable quantification of the likely errors in such angular vibration measurements. For measurements from the side of a polished-circular rotor, errors are around $1 \%$ for amplitude and $10 \mathrm{mrad}$ at integer orders affected by pseudo-vibration and around one-third of these levels elsewhere. For measurements from a rough rotor end face, errors will be similar at integer orders (from 2). Errors in the rotational speed measurement, required for postprocessing, must be minimised in order to limit errors up to 2 nd order while misalignments determine errors at around $0.4 \%$ amplitude and $4 \mathrm{mrad}$ phase at orders above 2 other than at the integer orders.
\end{abstract}

KEYWORDS: Laser vibrometry, angular, pitch, yaw, rotor, vibration measurement, surface roughness, misalignment, pseudo-vibration. 


\section{Introduction}

Vibration has long been acknowledged as the most effective measure of the condition of rotating machines [1],[2]. Translational vibration measurements dominate with widely available transducers including traditional piezo-electric accelerometers, newer MEMS-based devices and single beam laser vibrometers. Angular vibration measurements are also possible, either by combination of translational measurements, sometimes packaged for the specific purpose, or with dedicated devices [3]-[5]. With a wealth of important applications, methods for torsional vibration measurements are numerous and include optical sensing [6], toothed wheels [7],[8] and laser vibrometry [9]-[11]. With the exception of the torsional application, however, angular vibration measurements are much less commonplace than their translational counterparts.

Amongst the established methods of angular vibration measurement, laser vibrometry features prominently. This has been achieved with a scanning (single beam) laser vibrometer through densely spaced measurements in modal analysis [12] and using continuous scanning with dedicated postprocessing [13] but it is the parallel beam laser vibrometer that provides direct measurement of angular vibration on either non-rotating [14] or rotating [15] structures. This paper examines the use of parallel beam laser vibrometers for measurement of the $x$ - and $y$-components of angular vibration velocity, known as pitch and yaw respectively, directly from rotors. It follows and complements a recent study of radial vibration measurements directly from rotors using single beam laser vibrometers [16] in which the profound effect of surface roughness (or treatment) was explored in detail.

Remote, non-contact operation and insensitivity to target shape (for rough surfaces) are recognised as advantages of single and parallel beam laser vibrometers for general applications but these factors are especially valuable for rotor applications [15],[16]. Parallel beam laser vibrometers offer the additional advantage of insensitivity to translational vibrations, a factor of particular value in torsional vibration measurements on rotors and in pitch / yaw vibration measurements from the exposed end face of a rotor such as the crankshaft pulley of an engine [17]. For the rotor application, however, there are two significant problems to overcome. The first is pseudo-vibration, a periodic noise associated with the laser speckle phenomenon on rough surfaces and with variations in the target location from which collected light intensity is centred for polished surfaces [18]. The second is a cross-sensitivity to yaw in a pitch measurement and vice-versa, which affects all measurements in which there is some degree of diffuse scatter from the surface but which can only be reliably estimated for surfaces coated in retro-reflective tape. Since the angular vibration measurements of interest in this paper are effectively differential radial vibration measurements, this cross-sensitivity has the same basis as that observed in radial vibration measurements. Cross-sensitivity was not apparent in radial vibration measurements on polished-circular rotors and the reasons for this have already been articulated [16]. Parallel beam laser vibrometer measurements on polished-circular 
rotors have not been reported previously and this is part of the novelty of this paper. A focussed experimental investigation is presented.

Where cross-sensitivity does occur, a resolution algorithm has been developed [19] and its accuracy for the pitch and yaw vibration measurement application is explored, using computational simulation based on typical values, in this paper for the first time. The resolution algorithm is complemented by a second algorithm to correct for the effect of rotor speed fluctuations; its effectiveness is also explored for the angular vibration measurement application, again, for the first time. Finally, the measurement uncertainties associated with speed measurement error, measurement noise and inevitable instrument misalignment are all quantified individually and jointly (as would be the scenario in a typical realworld measurement) all for the first time in this paper. The overall aim of the paper is to provide practical guidance to the laser vibrometer practitioner in the form of recommended measurement configurations, to isolate certain intended angular motion components, with likely measurement uncertainties quantified for those configurations.

\section{Surface velocity and measured velocity}

The velocity measured by a parallel beam instrument in a rotor application incorporating full, six degree-of-freedom motion was first described by Bell [15]. More recently, a universal framework for modelling measured velocity has been proposed [20] and this will be used here, for the first time, to model measurements made with dual parallel beam instruments. An advantage of the new approach is the ease with which inevitable misalignments can be incorporated and this will set out in this section. The parallel beam models can be constructed conveniently as developments of the single beam models previously derived for radial vibration measurement from both polished-circular and rough rotors [16] as set out in the remainder of this section.

\subsection{Review of single beam models}

When the rotor surface is rough (i.e. the surface roughness is at least comparable with the laser wavelength), the measured velocity for a single beam measurement is written as follows:

$U_{m}=-\hat{b}_{i n} \cdot\left(\overrightarrow{V_{O^{*}}}+\vec{\omega} \times\left(\overrightarrow{r_{0}}-\overrightarrow{O O^{*}}\right)\right)$

in which $\hat{b}_{i n}$ is the direction of laser beam incidence, $\overrightarrow{V_{O^{*}}}$ is the velocity of a reference point fixed to the rotor centre-line, $\overrightarrow{r_{0}}$ is a vector defining an arbitrary known point along the beam and $\overrightarrow{O O^{*}}$ describes the displacement of reference point $O^{*}$ from its original position $O$ which also serves as the origin of the global coordinate system and is as shown in Figure 1a. Equation (1) holds when the 
scattering of light is diffuse such that the dominant contribution to the collected light originates from the geometric centre of the Gaussian laser beam as indicated by the upper ray within the laser beam in Figure $1 b$.

The angular velocity vector, $\vec{\omega}$, includes both angular vibration velocities around the three coordinate axes, $\left[\begin{array}{lll}\dot{\theta}_{x} & \dot{\theta}_{y} & \dot{\theta}_{z}\end{array}\right]^{7}$, and the continuous target rotation at angular frequency $\Omega$. This continuous rotation occurs around an axis, defined by the unit vector $\hat{z}_{R}$ as shown in Figure 1b, that is itself affected by the angular oscillations of the target. This is written in terms of an initial $z$-orientation modified by pitch and yaw vibration displacements, enabling a full expression for angular velocity to be written as:

$\vec{\omega}=\overrightarrow{\omega^{\prime}}+\Omega \hat{z}_{R}$

in which

$\overrightarrow{\omega^{\prime}}=\left[\begin{array}{lll}\hat{x} & \hat{y} & \hat{z}\end{array}\left[\begin{array}{lll}\dot{\theta}_{x} & \dot{\theta}_{y} & \dot{\theta}_{z}\end{array}\right]^{\top}\right.$

and

$$
\hat{z}_{R}=\left[\begin{array}{lll}
\hat{x} & \hat{y} & \hat{z}
\end{array}\left[y, \theta_{y}\right] x, \theta_{x}\left[\begin{array}{lll}
0 & 0 & 1
\end{array}\right]^{T}=\left[\begin{array}{lll}
\hat{x} & \hat{y} & \hat{z}
\end{array}\right]_{\cos \theta_{x} \sin \theta_{y}}-\sin \theta_{x} \quad \cos \theta_{x} \cos \theta_{y}\right]^{T}
$$

On combining equations (1) and (2a), it is the cross-product $\left(\Omega \hat{z}_{R} \times \overrightarrow{O O^{*}}\right)$ that introduces a crosssensitivity in the radial measurements to $y$-radial vibration in a measurement of $x$-radial velocity and vice-versa.

In a measurement from a polished-circular rotor, there will be a tendency for incident light that is not on a line passing directly through the centre of rotation to be reflected away from the vibrometer's collecting aperture. This represents a shift in the effective centre of the laser beam away from the geometric centre. Figure $1 \mathrm{~b}$ shows a second ray within the incident laser beam, incident on the displaced rotor (radius $R$ ) at point $P^{\prime \prime}$. This ray intersects the rotor centre-line at point $Q$ and, for a polished-circular rotor, it represents the part of the incident laser beam that instantaneously dominates the collected light intensity. For this case, the measured velocity is written as follows:

$U_{m} \approx-\hat{b}_{i n} \cdot\left(\overrightarrow{V_{O^{*}}}+\overrightarrow{\omega^{\prime}} \times\left(\overrightarrow{r_{0}}-\overrightarrow{O O^{*}}\right)\right)$ 
Polished-circular rotor measurements do not display the same cross-sensitivity encountered in rough rotor measurements and equation (3) shows how this has been eliminated; the equivalent crossproduct here is $\left(\overrightarrow{\omega^{\prime}} \times \overrightarrow{O O^{*}}\right)$ in which there is clearly no dependence on either $\Omega$ or $\hat{z}_{R}$.

\subsection{Extension to parallel beam models}

Extending the model to the parallel beam arrangement is straightforward in the new universal framework. Parallel beam instruments measure the differential velocity, $\Delta U_{m}$, between the two individual beams. Vectors for the arbitrary known points for each beam, $\overrightarrow{r_{01}}$ and $\overrightarrow{r_{02}}$, can be chosen, without any loss of generality, such that the vector $\vec{d}$, where $\vec{d}=\overrightarrow{r_{01}}-\overrightarrow{r_{02}}$, is perpendicular to both beams as shown in Figure 2.

For the rough rotor model, the measured differential velocity can then be written as:

$$
\Delta U_{m}=-\hat{b}_{i n} \cdot\left(\left(\overrightarrow{V_{O^{*}}}+\vec{\omega} \times\left(\overrightarrow{r_{01}}-\overrightarrow{O O^{*}}\right)\right)-\left(\overrightarrow{V_{O^{*}}}+\vec{\omega} \times\left(\overrightarrow{r_{02}}-\overrightarrow{O O^{*}}\right)\right)\right)=\left(\hat{b}_{i n} \times \vec{d}\right) \vec{\omega}
$$

while for the polished-circular rotor model, the differential velocity can be written:

$$
\Delta U_{m} \approx-\hat{b}_{i n} \cdot\left(\left(\overrightarrow{V_{O^{*}}}+\overrightarrow{\omega^{\prime}} \times\left(\overrightarrow{r_{01}}-\overrightarrow{O O^{*}}\right)\right)-\left(\overrightarrow{V_{O^{*}}}+\overrightarrow{\omega^{\prime}} \times\left(\overrightarrow{r_{02}}-\overrightarrow{O O^{*}}\right)\right)\right)=\left(\hat{b}_{i n} \times \vec{d}\right) \overrightarrow{\omega^{\prime}}
$$

The simplicity of the final expressions of equations $(4 a \& b)$ reveals several key attributes of the parallel beam measurement of angular velocity. Firstly, the insensitivity to target shape associated with the single beam rough rotor measurement is obviously retained and, secondly, insensitivity to translational vibration (i.e. no dependence on $\overrightarrow{V_{O^{*}}}$ or $\overrightarrow{O O^{*}}$ ) is also apparent in both measurements. Known points along the path of the beam are not required; measured velocity depends only on the orientation of the beams, the plane in which they coincide and their perpendicular separation.

Expression as an angular velocity is achieved by simply dividing the differential velocity by $|\vec{d}|$.

Isolation of the $x$-component of angular velocity (pitch) requires that neither $\hat{b}_{i n}$ nor $\vec{d}$ has an $x$ component. Practically, this means any beam arrangement in which the plane of the laser beams is parallel with the $y z$-plane is acceptable; this allows for incidence along the side of and on the face of the shaft. Similarly, to isolate the $y$-component of angular velocity (yaw) requires that neither $\hat{b}_{\text {in }}$ nor $\vec{d}$ has a $y$-component, in this case allowing for any beam arrangement in which the plane of the laser beams is parallel with the $x z$-plane; again, this includes incidence along the side of or on the face of the shaft. Finally, in the same way, to isolate the $z$-component of angular velocity (torsional or roll 
vibration) requires that neither $\hat{b}_{i n}$ nor $\vec{d}$ has a $z$-component, allowing for any beam orientation in which the plane of the laser beams is parallel with the $x y$-plane. Here the ideal alignment is on the side of a shaft only as a face measurement will always contain unwanted components of pitch and / or yaw vibration.

To write expressions for any laser beam orientation requires an initial (arbitrary) choice of orientation with desired final orientation achieved by an appropriate series of rotations. For example, Figure 3 shows an initial laser beam orientation of $-\hat{b}_{i n}=\hat{x}$, followed by a first rotation by $\beta$ around the $y$-axis and a second rotation by $\gamma$ around the $z$-axis. Figure 4 shows vector $\vec{d}$ based on the same initial beam orientation, i.e. with $-\hat{b}_{i n}=\hat{x}$, but with a first rotation by $\alpha$ around $\hat{x}$. The same rotations applied to beam orientation can subsequently be applied to $\vec{d}$. This approach will deliver totally general expressions but this paper is specifically concerned with the misalignments that inevitably accompany real-world measurements. Consequently, the initial orientation chosen is the ideal orientation with misalignments then added as follows: angular misalignment of $\vec{d}$ around the axis coinciding with the ideal laser beam incidence, followed by misalignment of both $\hat{b}_{i n}$ and $\vec{d}$ by rotations around the two orthogonal axes. This is a strategy that can be applied consistently for any orientation.

For a pitch measurement from the side of a shaft, the ideal alignments are $-\hat{b}_{i n}= \pm \hat{y}$ and $\vec{d}=\mp|\vec{d}| \hat{z}$ combined with a rotation around the $x$-axis of any value. Choosing the $-\hat{b}_{i n}=+\hat{y}$ option:

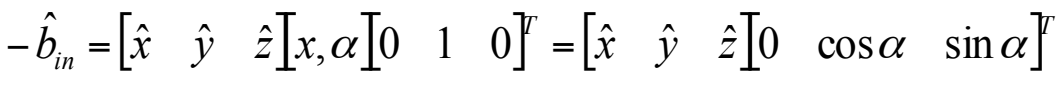

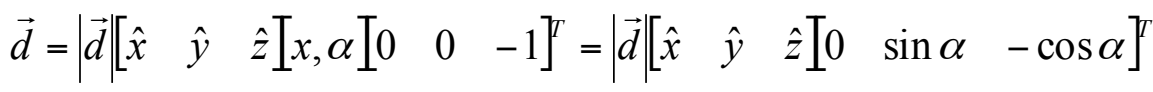

$$
\begin{aligned}
& \hat{b}_{i n} \times \vec{d}=|\vec{d}| \hat{x}
\end{aligned}
$$

While the individual vectors are affected by $\alpha$, their cross product is not unless misalignments are incorporated in which case:

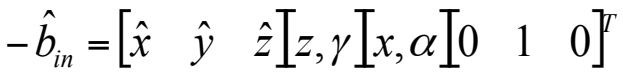

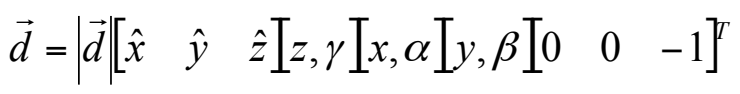

$$
\begin{aligned}
& \hat{b}_{i n} \times \vec{d} \approx \mid \vec{d}\left[\begin{array}{lllll}
\hat{x} & \hat{y} & \hat{z}] 1 & \gamma+\beta \sin \alpha & -\beta
\end{array}\right]^{T}
\end{aligned}
$$


Equation (6c) shows that minimising $\alpha$ helps to reduce the influence of misalignments. With this in mind (i.e. keeping $\alpha$ small), pitch measurements from the face of a shaft have ideal alignments of $-\hat{b}_{i n}= \pm \hat{z}$ and $\vec{d}= \pm|\vec{d}| \hat{y}$. Modified by misalignments, the $-\hat{b}_{i n}=+\hat{z}$ configuration is written as:

$-\hat{b}_{i n}=\left[\begin{array}{lll}\hat{x} & \hat{y} & \hat{z}\end{array} y, \beta\left[x, \alpha\left[\begin{array}{lll}0 & 0 & 1\end{array}\right]^{T} \approx\left[\begin{array}{lll}\hat{x} & \hat{y} & \hat{z}\end{array}\right] \beta\right.\right.$

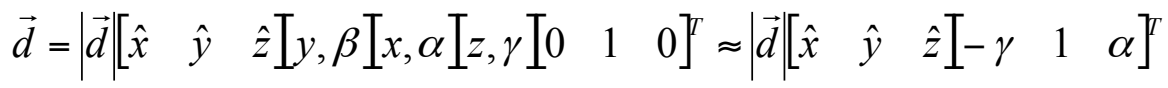

$\hat{b}_{i n} \times \vec{d} \approx|\vec{d}|\left[\begin{array}{lllll}\hat{x} & \hat{y} & \hat{z}\end{array} 1 \quad \gamma \quad-\beta\right]^{T}$

The approximations shown in equations (6c) and (7c) enable identification of critical misalignments but later simulations use full expressions and are without approximation.

The same considerations apply to yaw measurements. For a yaw measurement from the side of a shaft, the ideal alignments are $-\hat{b}_{i n}= \pm \hat{x}$ and $\vec{d}= \pm|\vec{d}| \hat{z}$. Choosing the $-\hat{b}_{i n}=+\hat{x}$ alternative and modifying for misalignments:

$$
\begin{aligned}
& -\hat{b}_{i n}=\left[\begin{array}{lll}
\hat{x} & \hat{y} & \hat{z}
\end{array}\right], \gamma\left[y, \beta[1 \quad 00 \quad 0]^{T}\right.
\end{aligned}
$$

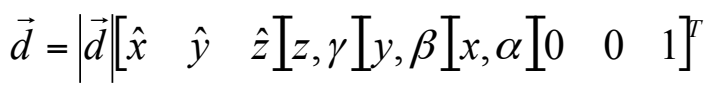

For a yaw measurement from the end face of a shaft, the ideal alignments are $-\hat{b}_{i n}= \pm \hat{z}$ and $\vec{d}=\mp|\vec{d}| \hat{x}$. Modified by misalignments, the $-\hat{b}_{i n}=+\hat{z}$ configuration is written as:

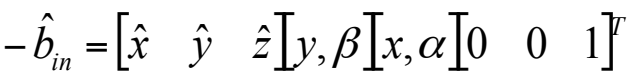

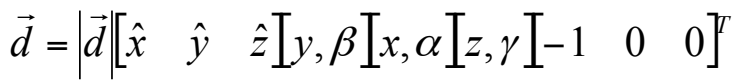

In the absence of misalignments, combining equation (6c) or (7c) with equations (4a) and (2a-c) and expressing as an angular velocity gives the pitch measurement as:

$$
\Omega_{m x}=\dot{\theta}_{x}+\Omega \cos \theta_{x} \sin \theta_{y} \approx \dot{\theta}_{x}+\Omega \theta_{y}
$$

Similarly combining equations ( $8 a \& b)$ or $(9 a \& b)$ with equations (4a) and (2a-c) gives the yaw measurement as: 
$\Omega_{m y}=\dot{\theta}_{y}-\Omega \sin \theta_{x} \approx \dot{\theta}_{y}-\Omega \theta_{x}$

This pair of equations for the measurement of pitch and yaw vibration shows the significant crosssensitivity to the orthogonal motion component in measurements when $\Omega \neq 0$. With the small angle approximation included, they are similar to those found in equivalently arranged radial vibration measurements [16] and can therefore be resolved for the parameters of interest in the same way. This resolution procedure will be described in section 4 .

The ideal alignments for measurements from the side of the rotor are also suitable for the polishedcircular rotor application. While the influence of misalignments prevails through identical expressions for beam geometry, equation (4b) results in absence of the cross-sensitivity that affects the rough rotor measurement. This has not been explored experimentally previously and will be confirmed in this paper. Provided that the plane of the rotor face is sufficiently perpendicular to the beam orientation, measurements will also be possible from a polished rotor face. Without the surface curvature that results in the change in the effective centre of the incident beam, equation (4a) should apply, however, and cross-sensitivity in the form of equations $(10 \mathrm{a} \& \mathrm{~b})$ should be found. This will be investigated for the first time in this paper.

\section{The effect of surface roughness and treatment on cross-sensitivity}

As shown in Figure 1b, for single beam measurements of radial vibration from the side of a rotor, a transition has been demonstrated in the effective centre-line of the beam from the line following the geometric centre of the laser beam for a rough rotor to a (nearly) parallel line passing through the rotor centre for a polished-circular rotor [16]. This same transition will occur for each of the individual beams in the parallel pair and this will be confirmed in this experimental investigation, concentrating on polished surfaces and surfaces coated in retro-reflective tape. This is in line with the key finding of the radial vibration study, which was that reliable measurements were only possible from such surfaces, and consistent with the overall aim of this study which is to provide practical guidance to the user.

The effect of vibration amplitude relative to beam diameter is also of interest and will be investigated. The Polytec OFV400 used in this study has three recommended stand-off distances: $200 \mathrm{~mm}, 400 \mathrm{~mm}$ (preferred) and $600 \mathrm{~mm}$, with corresponding beam diameters of 550 (predicted), 520 (measured) \& 605 (measured) $\mu \mathrm{m}$, respectively.

\subsection{Experimental investigation}

The cross-sensitivity ratio, $R$, has been defined in the radial vibration study [16]. With constant rotation speed, pitch vibration at frequency, $\omega_{v}$, and nominally zero yaw vibration, the equivalent 
cross-sensitivity ratio for this study is calculated based on pitch and yaw vibration measurements as follows:

$$
R=100 \frac{\omega_{v}}{\Omega} \frac{\left|F T\left[\Omega_{m y}\right]_{\omega_{v}}\right|}{\left|F T\left[\Omega_{m x}\right]_{\omega_{v}}\right|}
$$

where $F T[]_{\omega_{v}}$ signifies the Fourier transform of the velocity evaluated at frequency $\omega_{v}$. Angular vibration measurements on rotors with their surface coated with retro-reflective tape have been investigated previously [19] and will return a ratio of $100 \%$, in accordance with the theory presented in section 2.2. Measurements without cross-sensitivity would, conversely, return a ratio of zero.

\subsection{Experimental setup}

As shown in Figure 5, a small, unloaded, nominally-balanced test rotor is driven by a DC motor via a flexible belt. The rig has been specifically developed for this investigation and the rotor exhibits minimal self-induced vibration. Rotors with different surface roughness / treatment can be mounted in the rig. The entire rotor / DC motor assembly has a reasonable static balance so as to minimise the load on the electromagnetic shaker which excites a nominally sinusoidal angular vibration of the rotor through a pushrod and ball joint. Some harmonic distortion of the angular vibration occurs but this does not affect the analysis performed which is based on pitch and yaw measurements made at identical frequencies. Test rotors have diameter $15 \mathrm{~mm}$, length $30 \mathrm{~mm}$ and, as set out in Table 1, have roughness between $\mathrm{Ra} 11 \mathrm{~nm}$ and $\mathrm{Ra} 44 \mathrm{~nm}$ for side of rotor measurements, a value of Ra $6.5 \mathrm{~nm}$ for the rotor end face measurements and treatment with retro-reflective tape for both side and face measurements.

The experimental arrangement of the laser beams and the rotor for the measurement scenario in which the cross-sensitive (yaw) measurement is made from the side of the rotor is shown in Figure 5 while that for the measurement scenario in which the yaw measurement is made from the rotor end face is shown in Figure 6. Both measurement scenarios are also shown schematically for completeness in Figure 7. In both cases, the genuine (pitch) measurement was made from the side of the shaft; this was simply a matter of convenience. In both cases, careful alignment is important. For the rough rotor measurements, sufficient collected light intensity is relatively easy to achieve but ideal alignments as set out in section 2.2 are vital. Angular misalignments are minimised by maximising the measured amplitude in the pitch measurement and minimising it in the yaw measurement, without rotation. For polished-circular rotors, alignment as above is again required but is assisted by ensuring that the reflected stripes of light sit centrally over the vibrometer aperture, as shown in Error! Reference source not found.. With reference to equations ( $8 \mathrm{a} \& \mathrm{~b})$, minimisation of $\alpha$ is achieved by ensuring that the stripes are directly above one another, minimisation of $\beta$ requires the stripes to be centred on 
the front of the instrument and minimisation of $\gamma$ is achieved when the stripes pass across the points visible on the aperture and through which the laser beams emerge from the instrument.

The cross-sensitive (yaw) measurements were taken at each of the three stand-off distances while the genuine (pitch) measurement was always taken at $400 \mathrm{~mm}$ stand-off distance. Tests were conducted for a number of combinations of rotation frequency (up to $25 \mathrm{~Hz}$ ), vibration frequency (up to $37.5 \mathrm{~Hz}$ ) and vibration velocity (generally from 50 to $500 \mathrm{deg} / \mathrm{s}$ ); these levels are intended to be representative of those typical in 'real-world' measurements. Vibration frequencies were always nominally midway between integer multiples of rotation frequency to avoid coincidence with pseudo-vibration related components which are concentrated at integer multiples of rotation speed [18].

\subsection{Cross-sensitivity: effect of surface roughness, vibration displacement and beam diameter}

Attention was focussed on those rotors conforming to the polished-circular rotor model from the radial vibration study [16] $(\mathrm{Ra} \leq 50 \mathrm{~nm}$ and out-of-roundness $\leq 10 \mu \mathrm{m})$ with surfaces treated with retro-reflective tape as the rough rotor option, as listed in Table 1. Conformance to the polishedcircular model was also dependent on the vibration displacement remaining below a specific percentage of beam diameter. Manufacturing constraints continue to prevent a study of rotor out-ofroundness so the rotors chosen for the side measurements all have similar, low out-of-roundness, as also listed in Table 1.

Figure 9a shows the cross-sensitivity ratios calculated for the measurements taken from the side of the rotor at $400 \mathrm{~mm}$ stand-off for rotor A2 $(\mathrm{Ra} 11 \mathrm{~nm})$. When the laser beams are positioned equi-distant from the centre of the axis around which the pitch vibration occurs (dashed line), the cross-sensitivity ratio is low (around 10\%) but not as low as is desired for the assumption of no cross-sensitivity to be reliable. When either of the beams is aligned with the axis around which the pitch vibration occurs (round dot and square dot lines), much lower cross-sensitivity values are obtained at low displacements. With either of these two arrangements, the surface displacement at one of the beams is negligible and correspondingly larger at the second beam. Why this should lead to such a favourable outcome relative to the arrangement where there is some surface displacement at both beam incidences is not clear but the outcome that minimum cross-sensitivity ratio is found when one of the beams is aligned for minimum surface displacement was repeatable for this and for other rotors. This low cross-sensitivity is maintained until the largest of the surface displacements at either beam reaches approximately $50 \%$ of beam diameter, beyond which cross-sensitivity grows substantially.

The equivalent cross-sensitivity ratios for rotors B ( $\mathrm{Ra} 24 \mathrm{~nm}$ ) and C ( $\mathrm{Ra} 44 \mathrm{~nm}$ ) can be seen in Figure $9 \mathrm{~b}$, again for side of rotor measurements at $400 \mathrm{~mm}$ stand-off; the data for rotor A2 are repeated in this figure for completeness. For all three rotors, the data shown are for the arrangements where the "bottom" beam is aligned for minimum surface displacement. The higher roughness of 
rotors $\mathrm{B}$ and $\mathrm{C}$ results in a low cross-sensitivity ratio at low displacements but it is higher than for rotor A2, consistent with the equivalent findings in the radial vibration measurement study [16]. Rotor $\mathrm{C}$ has lower cross-sensitivity ratios than rotor B despite higher surface roughness but no special significance is being given to this. Single roughness values summarise complex variations in roughness and the key point is that cross sensitivity is higher for rotors with Ra of a few tens of $\mathrm{nm}$ compared to rotors with Ra of around $10 \mathrm{~nm}$. A second important finding for these less polished rotors is that surface displacements must now be limited to around $20 \%$ of beam diameter to keep cross-sensitivity ratios acceptably low.

Figure 10 shows the cross-sensitivity ratios for rotor A2 $(11 \mathrm{~nm})$ as a function of surface displacement for various stand-off distances. Two sets of data are shown for the $400 \mathrm{~mm}$ stand-off (520 $\mu \mathrm{m}$ beam diameter) set-up, one taken at the beginning of the set of experiments and the second taken after the $200(550 \mu \mathrm{m}$ beam diameter) and $600 \mathrm{~mm}$ ( $605 \mu \mathrm{m}$ beam diameter) datasets had been acquired, i.e. after significant experiment reconfiguration. As stand-off distance increases, the vibration amplitude at which sufficient light intensity is collected should decrease. This is apparent in the $605 \mu \mathrm{m}$ beam diameter data where cross-sensitivity ratios begin to rise at about half the value of surface displacement as a percentage of beam diameter as that which they do for the measurements made at lower stand-off distances. Very little difference is apparent, however, between the datasets for 200 $\mathrm{mm}$ and $400 \mathrm{~mm}$ stand-off. Once light is reflected beyond the vibrometer aperture, light collection by diffuse scatter dominates and cross-sensitivity ratios rise. Measurements under these circumstances are sometimes difficult to obtain and inconsistent data points such as the $600 \mathrm{~mm}$ stand-off data point for surface displacement between 70 and $80 \%$ beam diameter is an example of this.

For side of rotor measurements with retro-reflective tape treatment (rotor G2), the cross-sensitivity ratio was always close to $100 \%(100.9 \%(0.48 \%)$ - four measurements with vibration displacement as a percentage of beam diameter up to 10 were made) as expected. The same was true for measurements on the rotor face treated with retro-reflective tape (rotor J; 100.2\% $(0.54 \%)$ ). These are shown in Figure 11 alongside measurements from a polished end face (rotor $\mathrm{H}$ (Ra $6.5 \mathrm{~nm}$ )) which also shows cross-sensitivity close to $100 \%(97.5 \%(2.52 \%))$. This interesting outcome for the polished end face measurement scenario is a valuable finding, confirming the suggestion made in section 2.2 and presented for the first time in this paper.

\section{Resolution of vibration components in rough rotor measurements}

Resolution and correction algorithms, previously described [16] and demonstrated [19] for radial vibration measurements, can also be used with the pitch / yaw vibration in order to resolve the measured signals. In the (practically unlikely) absence of any fluctuations in the shaft rotation speed, the resolution algorithm alone enables complete resolution (apart from at the synchronous frequency). 
In the presence of speed fluctuations, iterations through the correction algorithm are applied to yield improved estimates of vibration amplitudes.

For the case of parallel beam instruments, the resolution algorithm is formulated in terms of the alternating components of the measured signals, $\tilde{\Omega}_{m x}$ and $\tilde{\Omega}_{m y}$, and is implemented on a frequencyby-frequency basis as follows:

$$
\begin{aligned}
& \dot{\Theta}_{x}\left(\omega_{n}\right)=W\left(\omega_{n}\right) F T\left[\tilde{\Omega}_{m x}-\bar{\Omega} \int_{0}^{t} \tilde{\Omega}_{m y} d t\right]_{\omega_{n}} \\
& \dot{\Theta}_{y}\left(\omega_{n}\right)=W\left(\omega_{n}\right) F T\left[\tilde{\Omega}_{m y}+\bar{\Omega} \int_{0}^{t} \tilde{\Omega}_{m x} d t\right]_{\omega_{n}}
\end{aligned}
$$

where $W\left(\omega_{n}\right)$ is a frequency dependent weighting factor given by:

$$
W\left(\omega_{n}\right)=\omega_{n}^{2} /\left(\omega_{n}^{2}-\bar{\Omega}^{2}\right)
$$

There is a subtlety in this application of the resolution algorithm to angular vibrations relative to its use for radial vibrations [16] in that, here, resolution requires the mean value of the shaft rotation speed, $\bar{\Omega}$, rather than the mean value of the total velocity around the $z$-axis, $\overline{\Omega_{\text {tot }}}\left(=\overline{\Omega+\dot{\theta}_{z}}\right)$, as is the case for radial vibrations. Practically, a significant effect is not expected.

Simulating equal amplitude, harmonic pitch and yaw radial vibrations with 36 random values of phase difference (in the range $-\pi$ to $\pi$ ) emphasises the significance of this cross-sensitivity and the importance of post-processing. Without misalignments or measurement noise, Figure 12a shows the apparent error in the measured velocity as a percentage of genuine velocity for the pitch measurement. This apparent error is a function of phase and the figure shows the maximum and minimum errors across the rotation order range. The mean error is not shown for clarity in the figure but it varies between approximately $1000 \%$ at very low orders to just below $10 \%$ around $10^{\text {th }}$ order. After resolution, mean errors are very small for amplitude and phase. The maximum and minimum amplitude errors after resolution are also shown in Figure 12a, confirming that resolution always reduce errors in this simulation which includes simultaneous pitch and yaw vibrations at the same order with amplitudes of $2.5 \mathrm{mrad} \mathrm{s}^{-1} / \mathrm{rad} \mathrm{s}^{-1}$. This corresponds to a $5 \mathrm{~mm} \mathrm{~s}^{-1}$ difference across beams with $8 \mathrm{~mm}$ separation at 2500 RPM. As shown in Figure 12b, the error data before resolution are independent of velocity amplitude but the errors after resolution grow with vibration amplitude, approximately in proportion to the square of the increase in vibration amplitude, because of the small angle approximation inherent in equations $(10 \mathrm{a} \& \mathrm{~b})$. Mean error (again not shown) is still at least 
several hundred times lower after resolution than that before resolution. Data for yaw vibration are very similar and so not shown for the analysis in this section.

At first order, $\omega_{n}=\bar{\Omega}$, the weighting function $W\left(\omega_{n}\right)$ is seen to be infinite and the bracketed functions in equations $(12 \mathrm{a} \& \mathrm{~b})$ evaluate to zero. The resolved velocity cannot be determined and all resolved velocities including Figure 12 do not show data points at first order. This is a fundamental limitation of the use of laser vibrometry for this application and not a consequence of the postprocessing method [19].

The resolved pitch / yaw velocities are affected by a fluctuating component in $\Omega$, i.e. $\Omega=\bar{\Omega}+\Delta \Omega$, such that the resolution algorithm outputs, given in equations (12a\&b), are no longer equal to just the intended measurement vibration velocities, $\dot{\Theta}_{x}\left(\omega_{n}\right)$ and $\dot{\Theta}_{y}\left(\omega_{n}\right)$, but include additional terms. Equations (12a\&b) are modified as follows:

$$
\begin{aligned}
& W\left(\omega_{n}\right) F T\left[\widetilde{\Omega}_{m x}-\bar{\Omega} \int_{0}^{t} \widetilde{\Omega}_{m y} d t\right]_{\omega_{n}}=\dot{\Theta}_{x}\left(\omega_{n}\right)+W\left(\omega_{n}\right) F T\left[\Delta \Omega \theta_{y}+\bar{\Omega} \int_{0}^{t} \Delta \Omega \theta_{x} d t\right]_{\omega_{n}} \\
& W\left(\omega_{n}\right) F T\left[\widetilde{\Omega}_{m y}+\bar{\Omega} \int_{0}^{t} \widetilde{\Omega}_{m x} d t\right]_{\omega_{n}}=\dot{\Theta}_{y}\left(\omega_{n}\right)-W\left(\omega_{n}\right) F T\left[\Delta \Omega \theta_{x}-\bar{\Omega} \int_{0}^{t} \Delta \Omega \theta_{y} d t\right]_{\omega_{n}}
\end{aligned}
$$

Compared to the radial vibration case, there is no sensitivity to radial offsets here but the error in the resolution algorithm outputs due to the combination of $\Delta \Omega$ and the vibration displacements remains and must again be minimised. Improved estimates of the resolved velocities, $\dot{\Theta}_{x, m+1}$ and $\dot{\Theta}_{y, m+1}$, can be obtained by (repeated) iterations through the correction algorithm, the outputs of which are given by:

$$
\begin{aligned}
& \dot{\Theta}_{x, m+1}\left(\omega_{n}\right)=\dot{\Theta}_{x, 1}\left(\omega_{n}\right)-W\left(\omega_{n}\right) F T\left[\Delta \Omega \theta_{y, m}+\bar{\Omega} \int_{0}^{t} \Delta \Omega \theta_{x, m} d t\right]_{\omega_{n}} \\
& \dot{\Theta}_{y, m+1}\left(\omega_{n}\right)=\dot{\Theta}_{y, 1}\left(\omega_{n}\right)+W\left(\omega_{n}\right) F T\left[\Delta \Omega \theta_{x, m}-\bar{\Omega} \int_{0}^{t} \Delta \Omega \theta_{y, m} d t\right]_{\omega_{n}}
\end{aligned}
$$

The improved estimates are, as can be observed, derived from the first estimates of the resolved velocities (i.e. the resolution algorithm outputs), $\dot{\Theta}_{x, 1}$ and $\dot{\Theta}_{y, 1}$, and the $m$ th estimate of the resolved angular vibration displacements in the time domain (with the exception of the synchronous frequency component), $\theta_{x, m}$ and $\theta_{y, m}$. 
The effect of speed fluctuation on the resolved outputs is demonstrated in Figure 13. Equal amplitude, harmonic pitch and yaw vibrations with 16 random values of phase difference, combined with 16 independent broadband speed variations (RMS level of $2 \%$ of rotation speed), are simulated. Figure 13a shows that the RMS error in resolved amplitude is between $0.1 \%$ and $1 \%$ at lower orders but small elsewhere. As shown in Figure 13b, the corresponding phase difference error is in the region of 1 to $10 \mathrm{mrad}$ at lower orders, falling to $0.1 \mathrm{mrad}$ at higher orders. Error increases as the RMS broadband speed variation increases.

After a first iteration through the correction algorithm $(m=1)$, Figure 14 shows how the error across the order range is reduced to around $3 \%$ of its uncorrected level for both amplitude and phase difference. After a further iteration, this figure shows that there is a further reduction to around $0.15 \%$ above first order. As in the case of radial vibration measurements, there is little further improvement with further iterations and routine addition of two iterations through the correction algorithm is, therefore, recommended as a good compromise between error reduction and post-processing time. Smaller reductions are seen below first order for the second iteration. Here errors are dominated by the inherent small angle approximation as seen in Figure 13a.

\section{Simulation}

This section presents simulations that demonstrate the relative effect of each source of error in rough and polished-circular rotor measurements. The error calculated at each rotation order is a consequence of simultaneous pitch and yaw vibrations at that same order with amplitudes of $2.5 \mathrm{mrad} \mathrm{s}^{-1} / \mathrm{rad} \mathrm{s}^{-1}$, unless stated otherwise. In all cases, realistic values for misalignments (see Appendix A) are used based on the authors' experience but the reader must be mindful that predicted errors are obviously very dependent on these chosen values. The paper has, however, presented the full theoretical basis to these simulations, enabling another investigator to model their specific scenarios and predict likely error levels.

Generally, only pitch error spectra are presented as the corresponding yaw data are identical. In addition, for the most intensive simulations, the resolution in the plots is made finer in the more rapidly changing regions of the spectra, i.e. close to the synchronous frequency, and left coarser elsewhere. Only 'good' setups contribute to the data presented; in real measurements excessive DC angular velocity is indicative of angular misalignment and is therefore routinely minimised. From the authors' experience, DC angular velocity can be reduced to levels around $1 \%$ of rotation speed. Simulations include a check to exclude individual setups that would be rejected in real measurements.

The effect of pseudo-vibration is shown in Figure 15. In simulated measurements from the side of a rotor, 32 random values of phase difference between the radial vibrations (in the range $-\pi$ to $\pi$ ) are 
combined with 64 independent measurement noises (with RMS levels of $0.3 \mathrm{mrad} / \mathrm{s} / \mathrm{rad} / \mathrm{s}$ over the first 10 orders for the rough rotor and a quarter of this level for the polished-circular rotor [16]), equating to a total of 2048 scenarios. For the rough rotor simulation, amplitude errors of around 5\% appear at the integer orders where periodic speckle noise occurs and $0.1 \%$ to $0.01 \%$ elsewhere. Phase difference errors of around $60 \mathrm{mrad}$ appear at the integer orders and 1 to $10 \mathrm{mrad}$ elsewhere. The polished-circular rotor simulation reflects the lower noise levels encountered while the advantage of there being no need for resolution brings significant benefit around the synchronous frequency. In both cases, errors change in inverse proportion to any change in vibration amplitudes i.e. a tenfold increase in vibration amplitude results in a tenfold decrease in errors. Measurements on the end face of a rotor result in lower noise levels. This is advantageous in the case of the rough rotor where pseudo-vibration errors are roughly one-tenth of those shown in Figure 15. It is less advantageous for the polished-circular rotor because cross-sensitivity is encountered in end-face measurements requiring resolution.

Figure 16 shows the effect of typical instrument misalignments. 84035 'good' misaligned setups are simulated (from initial consideration of 117649 setups) with 16 random values of phase difference between the radial vibrations, producing over 1.3 million scenarios. For the rough rotor simulation, amplitude errors of around $0.3 \%$ appear at frequencies away from synchronous, rising to $3 \%$ close to synchronous. Similarly, phase errors of around $3 \mathrm{mrad}$ appear at frequencies away from synchronous, rising to $60 \mathrm{mrad}$ close to synchronous. Comparable data for the polished-circular rotor show expected error levels at $0.3 \%$ for amplitude and $3 \mathrm{mrad}$ for phase. Vibration amplitude does not affect these levels. Angular misalignments around $y$ - and $z$-axes are critical in the pitch measurement, as indicated in equation (6c). Correspondingly, angular misalignments around $x$ - and $z$-axes are critical in the yaw measurement.

Speed measurement error is the subject of Figure 17. Errors from $-2.5 \%$ to $2.5 \%$, in steps of $0.5 \%$ are considered in combination with 32 random values of phase difference between the radial vibrations (a total of 352 scenarios). This error is unique to the rough rotor measurement; amplitude error is close to $2 \%$ at low frequencies, rising to $20 \%$ near synchronous before falling to around $1 \%$ at second order and $0.1 \%$ by tenth order while phase error rises from $1 \mathrm{mrad}$ at very low frequency to $100 \mathrm{mrad}$ near synchronous before falling to less than $10 \mathrm{mrad}$ at second order and almost $1 \mathrm{mrad}$ at tenth order. There is no effect of vibration amplitude on this error.

In the presence of pitch and yaw vibrations only, these three sources of error are dominant. Figure 18 shows their combination (16 independent measurement noises, 9375 misaligned setups, speed errors from $-2.5 \%$ to $2.5 \%$ in steps of $0.5 \%$ and 16 phases between the angular vibrations resulting in over 26 million scenarios). Speckle noise dominates the rough rotor measurement at integer orders from 2 upwards. Away from the integer orders, speed measurement error dominates up to around fifth order 
after which misalignments become the significant effect. Errors associated with the speed variations considered in Figure 13 and Figure 14 are at least an order of magnitude lower than these levels and so are negligible. Nonetheless, this simulation was repeated for the same set of 26 million scenarios but with the addition of four iterations around the correction algorithm which confirmed that there are no adverse effects on these error levels associated with its use. For the polished-circular rotor measurement, pseudo-vibration dominates at integer orders with misalignment determining error levels elsewhere.

\section{Conclusions}

This paper has combined an experimental study of the cross-sensitivity encountered in parallel beam laser vibrometry measurements of rotor pitch and yaw vibration with a quantitative evaluation of measurement errors based on simulation. The evaluation of the effects of misalignments, for both rough and polished-circular rotors, was made possible by a recently developed framework for a comprehensive mathematical prediction of measured velocity that is without approximation [18].

From this study, it can be concluded that rotors with surface roughness up to $50 \mathrm{~nm}$ and out-ofroundness below $10 \mu \mathrm{m}$ adequately satisfy the polished-circular rotor definition (side of shaft measurement), provided vibration displacement does not exceed a specific threshold related to beam diameter. For a rotor with $\mathrm{Ra}$ around $10 \mathrm{~nm}$, measurements are straightforward provided that surface displacement at either beam location does not exceed $50 \%$ of beam diameter. For rotors with Ra up to $50 \mathrm{~nm}$ the corresponding figure is reduced to around $20 \%$ of beam diameter. In both cases there is an advantage if surface displacement can be minimised at one of the beams. Measurements are also possible on the polished end face of a rotor but the absence of surface curvature makes crosssensitivity $100 \%$. Resolution is then necessary in the same way as for measurements on the side or end face of a rotor with retro-reflective tape surface treatment, which satisfies the rough rotor definition.

End face measurements are advantageous from the point of view of minimising errors due to pseudovibration. These factors combine to make recommendations to the vibration user straightforward. If measurements can be made from the side of a rotor that is polished and circular then low noise measurements without cross-sensitivity can be made. If this not possible then end face (or flange) measurements with the surface coated in retro-reflective tape are the next best option. There is no advantage to measurement from a polished end face which will also be affected by the temperamental nature of measurements made on a rotating polished surface. End face measurements with retroreflective tape will be tolerant of shape variations but will require the use of resolution and correction algorithms. 
The essential requirement for post-processing of the rough rotor measurement has been demonstrated quantitatively, as has the effectiveness of the resolution algorithm. In the presence of speed fluctuations, the value of a complementary correction algorithm has also been confirmed and two iterations through the algorithm are now recommended wherever speed fluctuation (including torsional vibration) is present.

Measurement errors can be readily predicted for any specific measurement scenario. For equal amplitude pitch / yaw vibrations, data have been generated for typical misalignments, measurement noise and speed measurement errors to provide typical error levels for the user to have in mind when interpreting data from such measurement scenarios. For measurements from the side of a polishedcircular rotor, errors are around $1 \%$ for amplitude and $10 \mathrm{mrad}$ at integer orders and around one-third of these levels elsewhere. For measurements from a rotor end face treated with retro-reflective tape, errors will be similar at integer orders (from 2). Speed measurement errors must be minimised to limit errors up to $2 \mathrm{nd}$ order and misalignments determine errors at around $0.4 \%$ amplitude and $4 \mathrm{mrad}$ phase at orders above 2 other than at the integer orders.

This study of the effects of surface roughness, instrument misalignments and measurement noise delivers an essential practical guide for the laser vibrometer user. 


\section{Appendix A: Misalignment values used in simulations}

For side of rotor measurements

Pitch:

misalignment around $x$-axis takes values from $-0.6^{\mathrm{O}}$ to $0.6^{\mathrm{O}}$ in steps of $0.2^{\mathrm{O}}$

misalignments around $y$ - and $z$-axes take values from $-0.3^{\mathrm{O}}$ to $0.3^{\mathrm{O}}$ in steps of $0.1^{\mathrm{O}}$

Yaw:

misalignments around $x$ - and $z$-axes take values from $-0.3^{\mathrm{O}}$ to $0.3^{\mathrm{O}}$ in steps of $0.1^{\mathrm{O}}$

misalignment around $y$-axis takes values from $-0.6^{\mathrm{O}}$ to $0.6^{\mathrm{O}}$ in steps of $0.2^{\mathrm{O}}$ 


\section{REFERENCES}

[1] J.S. Mitchell, Vibration analysis - its evolution and use in machinery health monitoring. Society of Environmental Engineers Symposium on Machine Health Monitoring, Imperial College, London, England (1975).

[2] A. Muszynska, Rotordynamics, Vol. 188 CRC Press 2005.

[3] P.C. Rymers, Angular-vibration measurement, Exp. Techs. 6(3) (2008) 1-3.

[4] S.J. Ovaska and S. Valiviita, Angular acceleration measurement: a review, Proc. of IEEE Instr. Meas. Tech. Conf. (1998) 875-880.

[5] H.J. Ahn and S. Jeon, Effects of the rotor obliquity on the cylindrical capacitive sensor, Meas. Sci. Tech. 22(4) (2011) 045207.

[6] P. Wang, P. Davies, J.M. Starkey and R.L. Routson, A torsional vibration measurement system, Trans. IEEE Instr. Meas. 41(6) (1992) 803-807.

[7] J. Williams, Improved methods for digital measurement of torsional vibration, SAE, Paper 962204 (1996).

[8] T. Addabbo, A. Fort, R. Biondi, S. Cioncolini, M. Mugnaini, S. Rocchi, and V. Vignoli, Measurement of angular vibrations in rotating shafts: effects of the measurement setup nonidealities, Trans. IEEE Instr. Meas. 62(3) (2013) 532-543.

[9] N.A. Halliwell, C.J.D. Pickering and P.G. Eastwood, Laser torsional vibrometer: a new instrument, J. Sound Vib. 93(4) (1984) 588-592.

[10] N.A. Halliwell, The laser torsional vibrometer: a step forward in rotating machinery diagnostics, J. Sound Vib., 190(3) (1996) 399-418.

[11] L. Xiang, S. Yang and C. Gan, Torsional vibration measurements on rotating shaft system using laser Doppler vibrometer, Opts. Las. Eng. 50(11) (2012) 1596-1601.

[12] M.J. Ratcliffe and N.A.J. Lieven, Measuring rotational degrees of freedom using a laser Doppler vibrometer, J. Vib. Acous. 122(1) (2000) 12-20.

[13] A.B. Stanbridge and D.J. Ewins, Measurement of translational and angular vibration using a scanning laser Doppler vibrometer, Shock Vib. 3(2) (1996) 141-152.

[14] N.A. Halliwell, A. Hocknell and S.J. Rothberg, On the measurement of angular vibration displacements: a laser tiltmeter, J. Sound Vib. 208(3) (1997) 497-500.

[15] J.R. Bell and S.J. Rothberg, Rotational vibration measurements using laser Doppler vibrometry: comprehensive theory and practical application, J. Sound Vib. 238(4) (2000) 673-690.

[16] S.J. Rothberg, B.J. Halkon, M. Tirabassi and C. Pusey, Radial vibration measurements directly from rotors using laser vibrometry: the effects of surface roughness, instrument misalignments and pseudo-vibration, Mech. Syst. Sig. Proc. 33(11) (2012) 109-131. 
[17] B.J. Halkon and S.J. Rothberg, Automatic post-processing of laser vibrometry data for rotor vibration measurements, Proc. IMechE Eighth Int. Conf. Vibs. Rot. Mach., Swansea, UK (2004) 215-230.

[18] P. Martin and S.J. Rothberg, Pseudo-vibration sensitivities for commercial laser vibrometers, Mech. Syst. Sig. Process. 25 (2011) 2753-2765.

[19] B.J. Halkon and S.J. Rothberg, Rotor vibration measurements using laser Doppler vibrometry: essential post-processing for resolution of radial and pitch / yaw vibrations, Trans. ASME J. Vib. Acoust. 128 (2006) 8-20.

[20] S.J. Rothberg and M. Tirabassi, A universal framework for modelling measured velocity in laser vibrometry with applications, Mech. Syst. Sig. Proc. 26(1) (2012) 141-166. 


\section{Table captions}

Table 1. Roughness and out-of-roundness of rotor samples.

\section{Figure captions}

Figure 1. Laser beam orientation showing a) the incident point on the displaced rotor and b) the points used in the vector polygons.

Figure 2. Parallel laser beams incident on a vibrating rotor.

Figure 3. Laser beam orientation, defining angles $\beta$ and $\gamma$.

Figure 4. Orientation of parallel beam separation, defining angle $\alpha$.

Figure 5. Experimental arrangement for which the cross-sensitive measurement was taken from the side of the rotor also showing the rotor rig assembly.

Figure 6. Experimental arrangement for which the cross-sensitive measurement was taken from the end face of the rotor.

Figure 7. Laser beams arrangement relative to rotor for side and end face cross-sensitivity measurements; in both cases the intended, genuine measurement is the $x$-angular (pitch).

Figure 8. Back-scattered light on front of vibrometer for a polished-circular rough rotor.

Figure 9. Effect of displacement amplitude ( $520 \mu \mathrm{m}$ beam diameter $-400 \mathrm{~mm}$ stand-off) for rotor A2 $(\mathrm{Ra} 11 \mathrm{~nm})$ for side of rotor measurements; also showing the impact of beam alignment relative to oscillation centre.

Figure 10. Effect of stand-off distance (and therefore beam diameter) for rotor A2 (Ra $11 \mathrm{~nm}$ ) for side of rotor measurements.

Figure 11. Effect of displacement amplitude (520 $\mu \mathrm{m}$ beam diameter, $400 \mathrm{~mm}$ stand-off) for rotor end face measurements.

Figure 12. Maximum and minimum errors in measured angular velocity prior to resolution (dashed lines) and after resolution (solid lines) a) $2.5 \mathrm{mrad} \mathrm{s}^{-1} / \mathrm{rad} \mathrm{s}^{-1}$ b) $25 \mathrm{mrad} \mathrm{s}^{-1} / \mathrm{rad} \mathrm{s}^{-1}$.

Figure 13. RMS error in resolved angular velocity as a result of broadband speed variation; a) pitch (solid line) and yaw (dashed line) amplitudes and b) phase difference.

Figure 14. Relative error in resolved velocity following $m$ iterations through the correction algorithm (*) $m=1$, solid line $m=2,(+) m=4$; a) pitch amplitude and b) phase difference.

Figure 15. RMS error in resolved angular velocity as a result of pseudo-vibration for polished-circular (solid line) and rough $(*)$ rotor model; a) pitch amplitude and b) phase difference. 
Figure 16. RMS error in resolved angular velocity as a result of misalignments for polished-circular (solid line) and rough $(*)$ rotor model; a) pitch amplitude b) phase difference.

Figure 17. RMS error in resolved angular velocity as a result of speed measurement error for rough rotor model; a) pitch amplitude b) phase difference.

Figure 18. RMS error in resolved angular velocity as a result of the combined effects of pseudovibration, misalignments and speed measurement error for polished-circular $\left(^{*}\right)$ and rough $(+)$ rotor model; a) pitch amplitude b) phase difference. 


\section{Tables}

Table 1. Roughness and out-of-roundness of rotor samples.

\begin{tabular}{|l|l|l|l|}
\hline $\begin{array}{l}\text { Rotor } \\
\text { ID }\end{array}$ & $\begin{array}{l}\text { Measurement } \\
\text { location }\end{array}$ & $\begin{array}{l}\text { Roughness Ra } \\
(\mathbf{n m})\end{array}$ & $\begin{array}{l}\text { Out-of-roundness } \\
(\boldsymbol{\mu} \mathbf{m})\end{array}$ \\
\hline A2 & Side & 11 & 6.7 \\
\hline B & Side & 24 & 4.5 \\
\hline C & Side & 44 & 6.5 \\
\hline G2 & Side & Retro-reflective tape & n/a \\
\hline H & End face & 6.5 & n/a \\
\hline J & End face & Retro-reflective tape & n/a \\
\hline
\end{tabular}


Figures
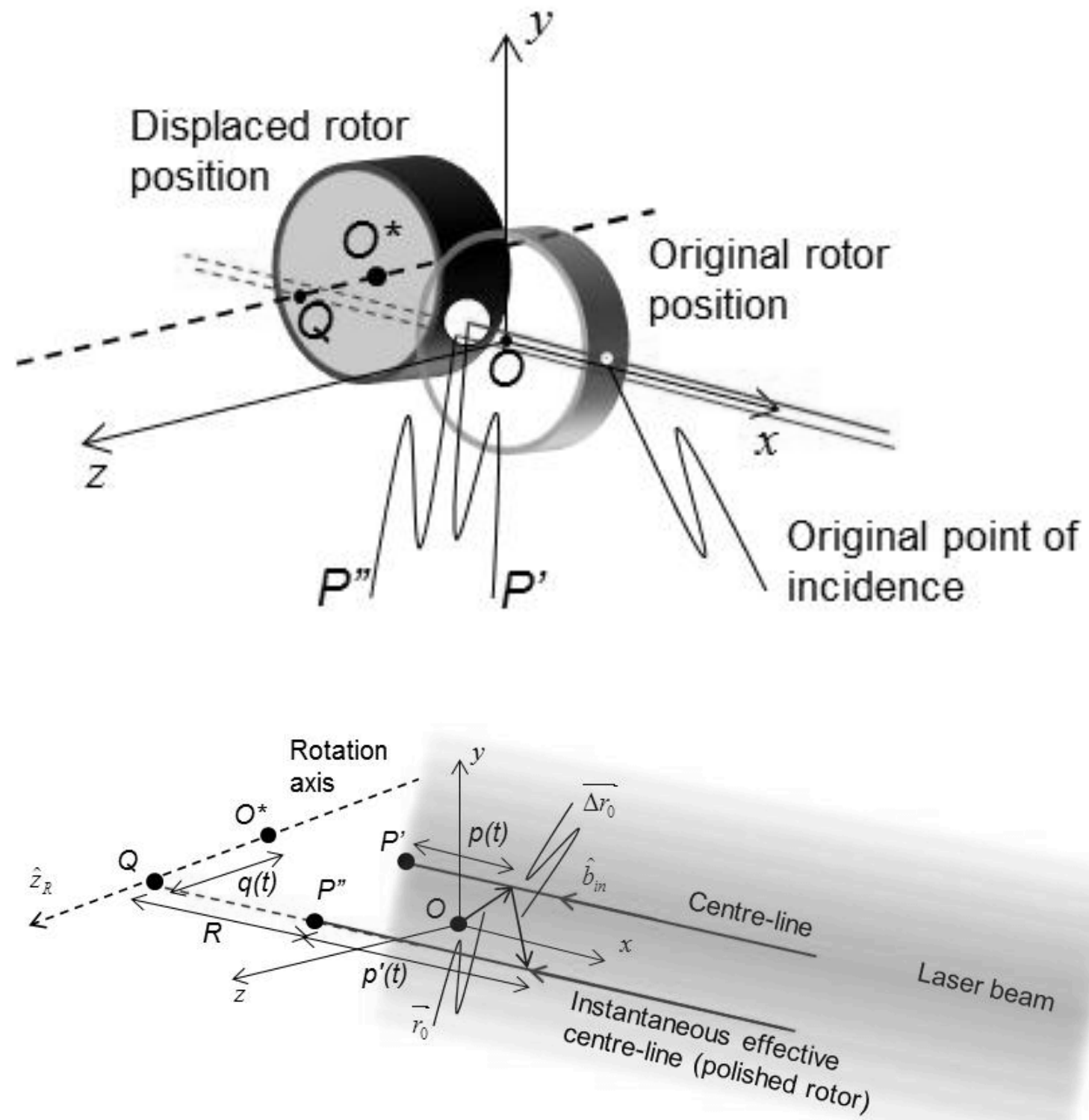

Figure 1. Laser beam orientation showing a) the incident point on the displaced rotor and b) the points used in the vector polygons. 


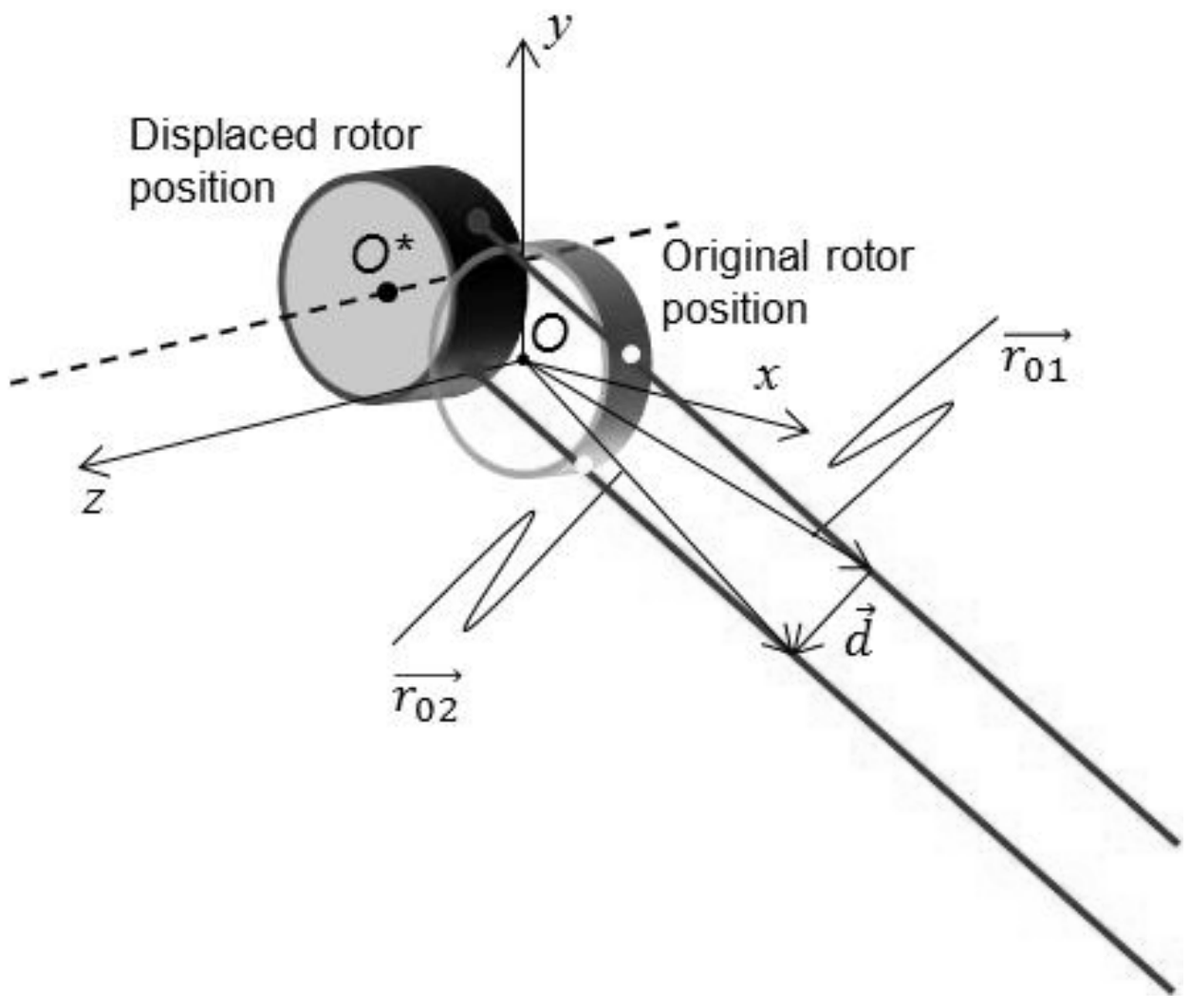

Figure 2. Parallel laser beams incident on a vibrating rotor. 


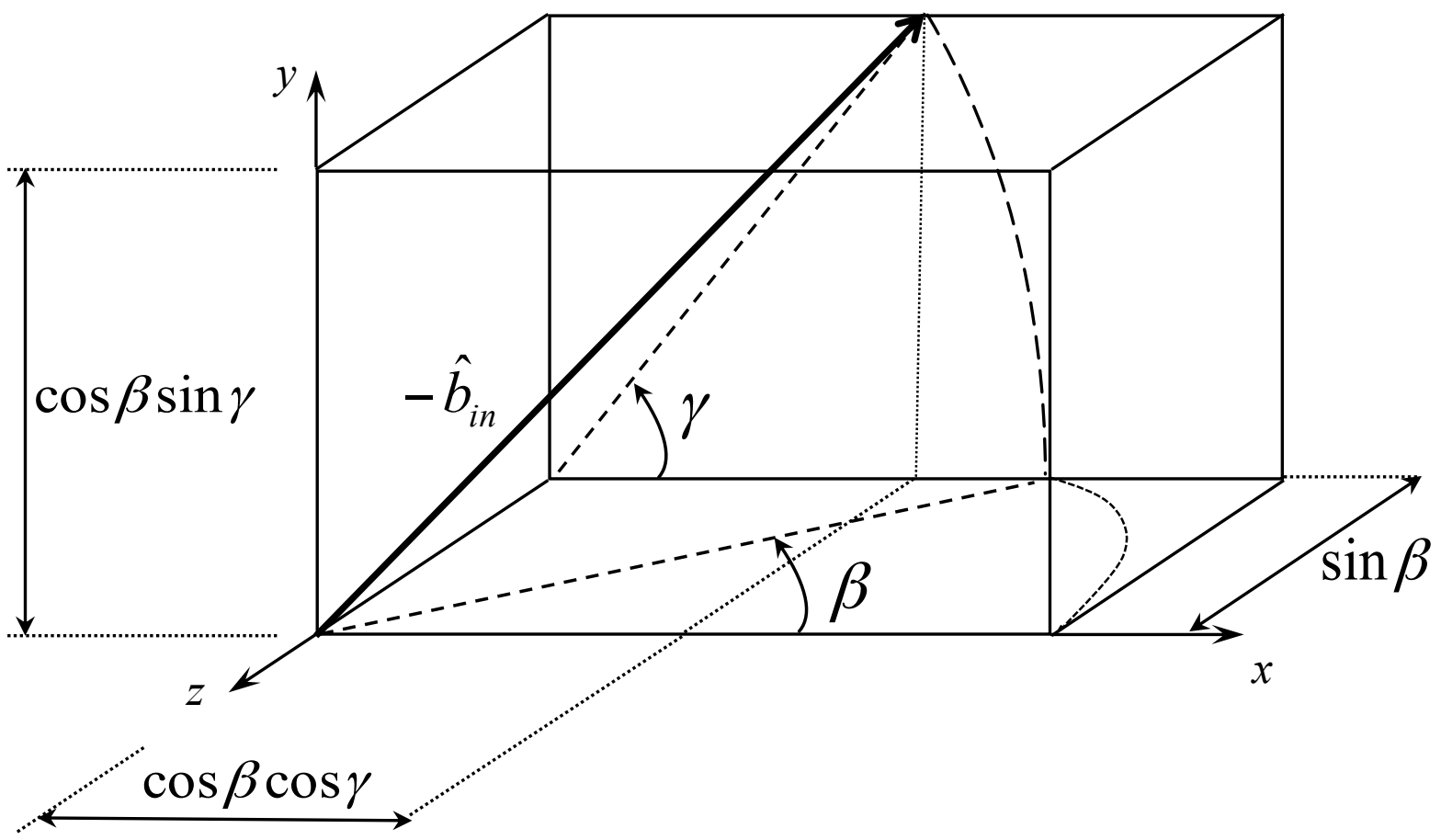

Figure 3. Laser beam orientation, defining angles $\beta$ and $\gamma$. 


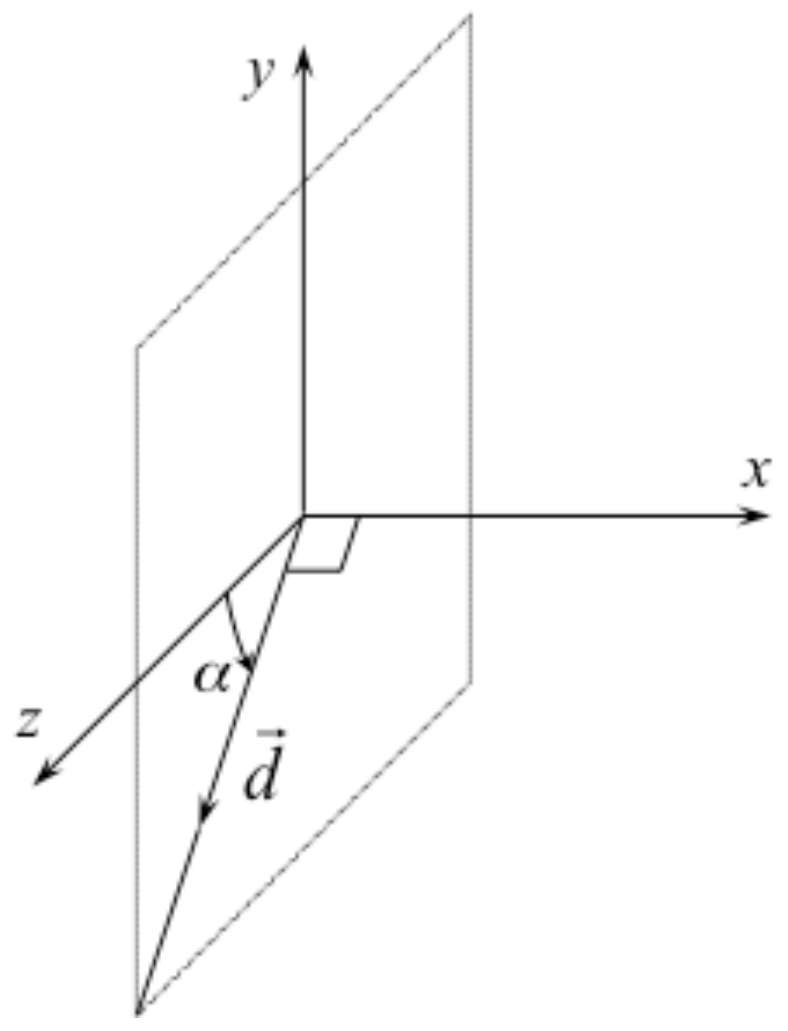

Figure 4. Orientation of parallel beam separation, defining angle $\alpha$. 


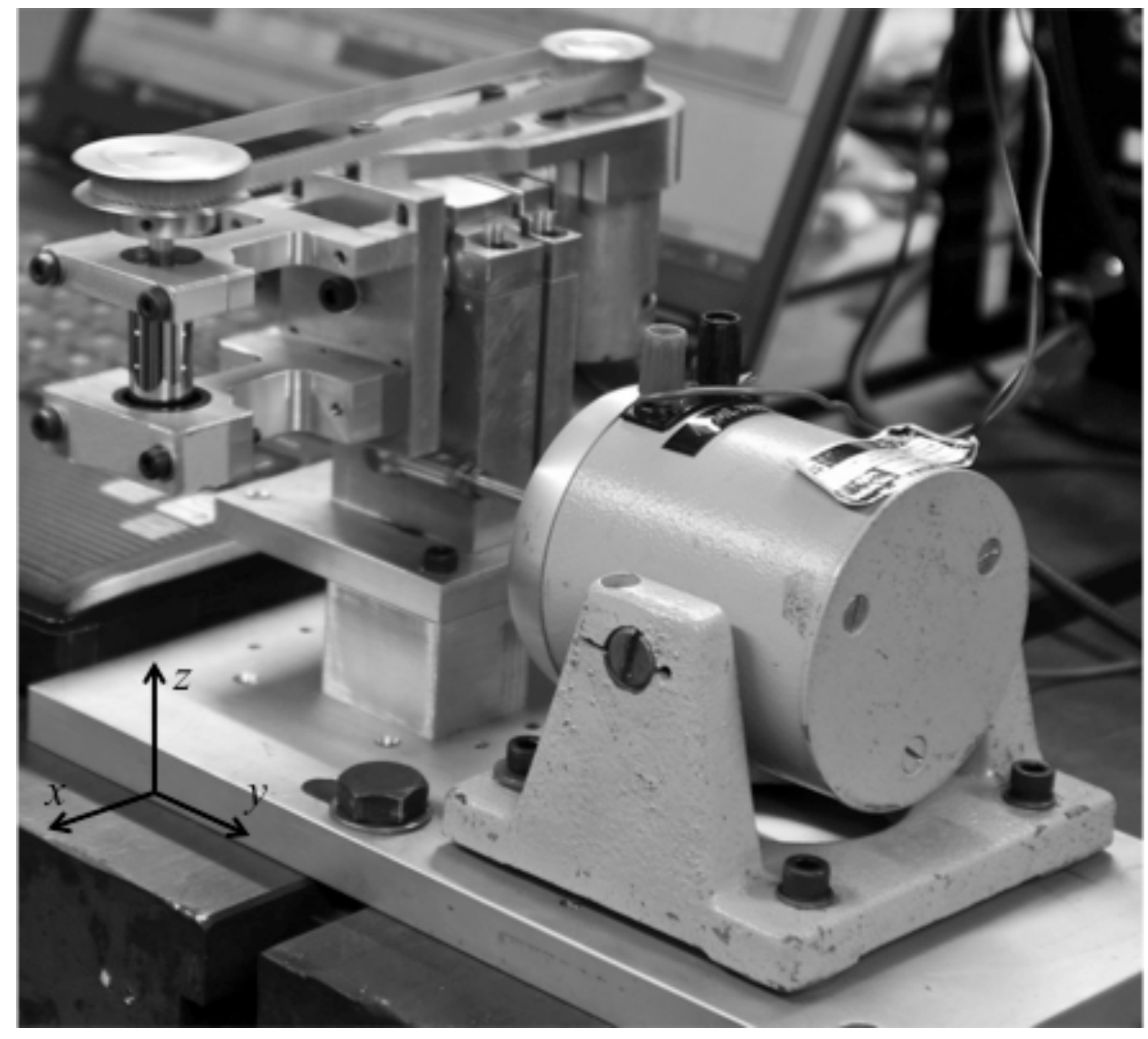

Figure 5. Experimental arrangement for which the cross-sensitive measurement was taken from the side of the rotor also showing the rotor rig assembly. 


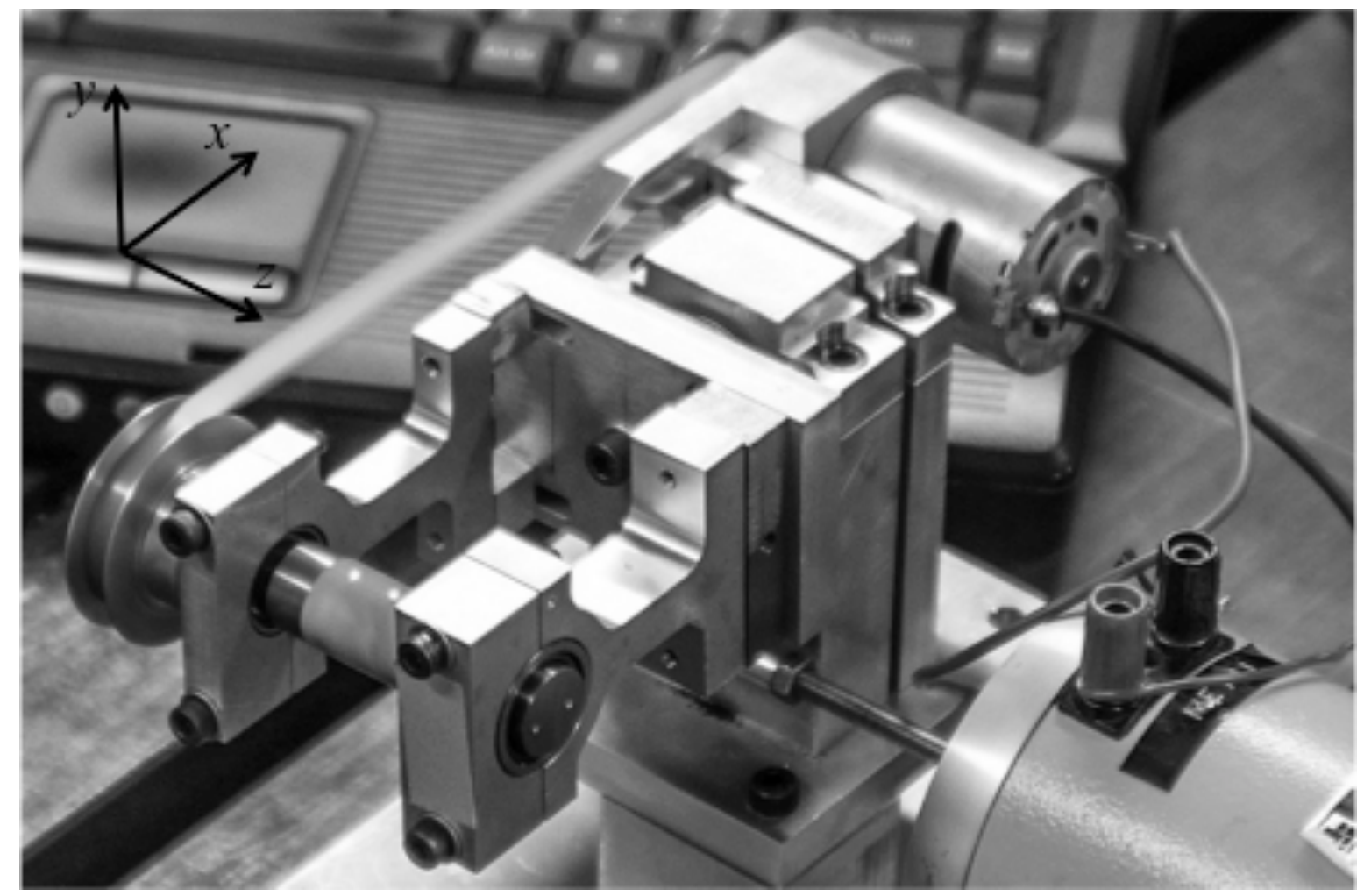

Figure 6. Experimental arrangement for which the cross-sensitive measurement was taken from the end face of the rotor. 

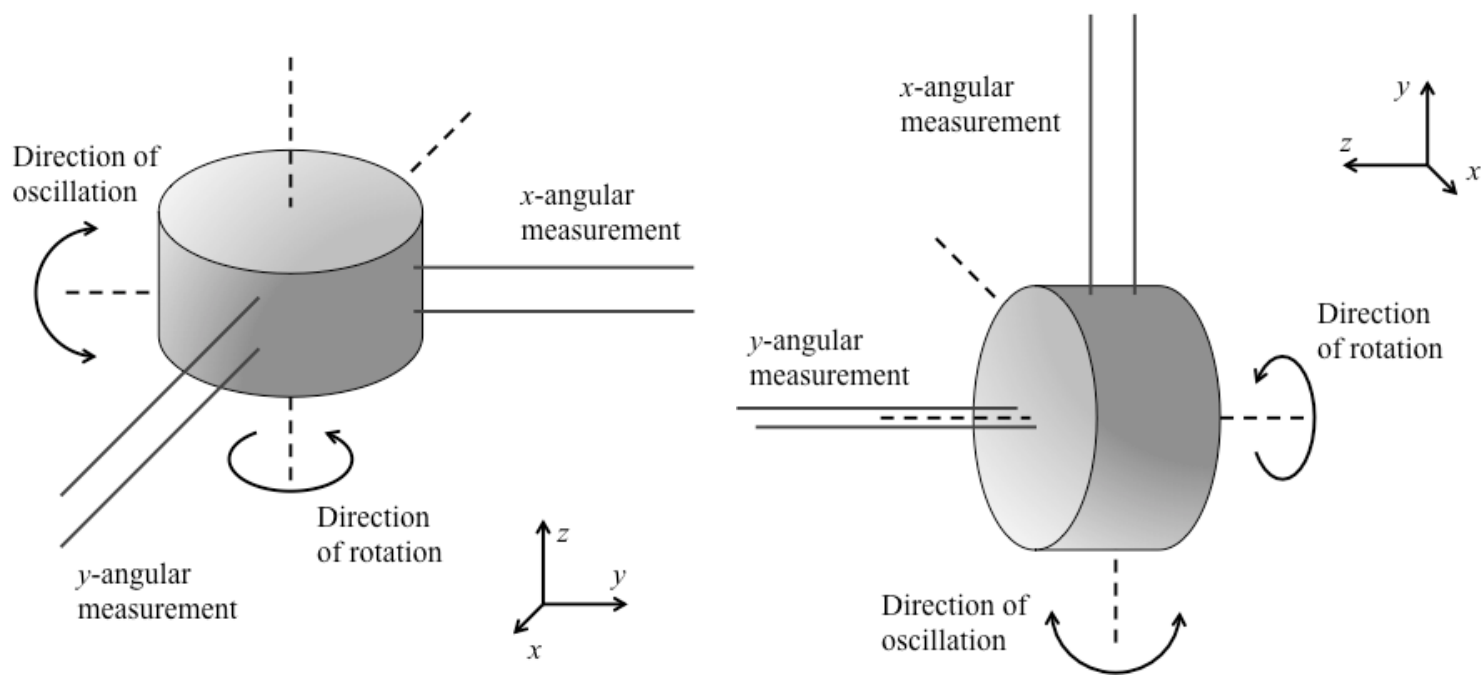

Figure 7. Laser beams arrangement relative to rotor for side and end face cross-sensitivity measurements; in both cases the intended, genuine measurement is the $x$-angular (pitch). 


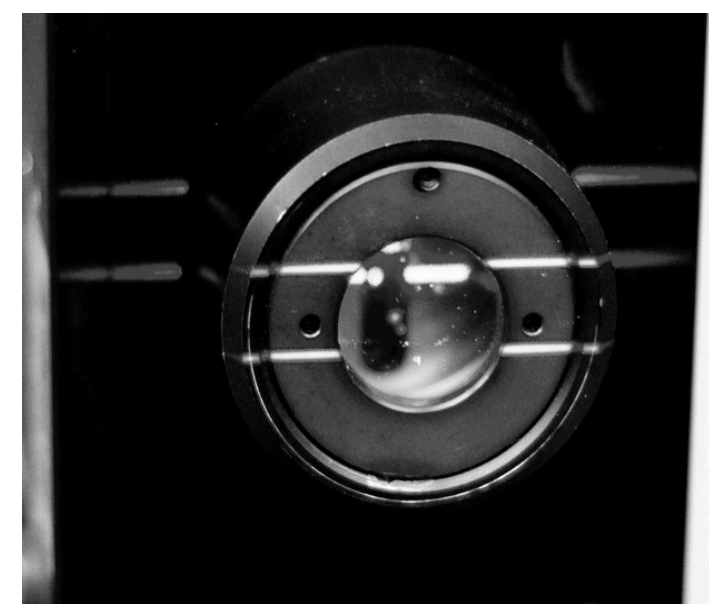

Figure 8. Back-scattered light on front of vibrometer for a polished-circular rough rotor. 

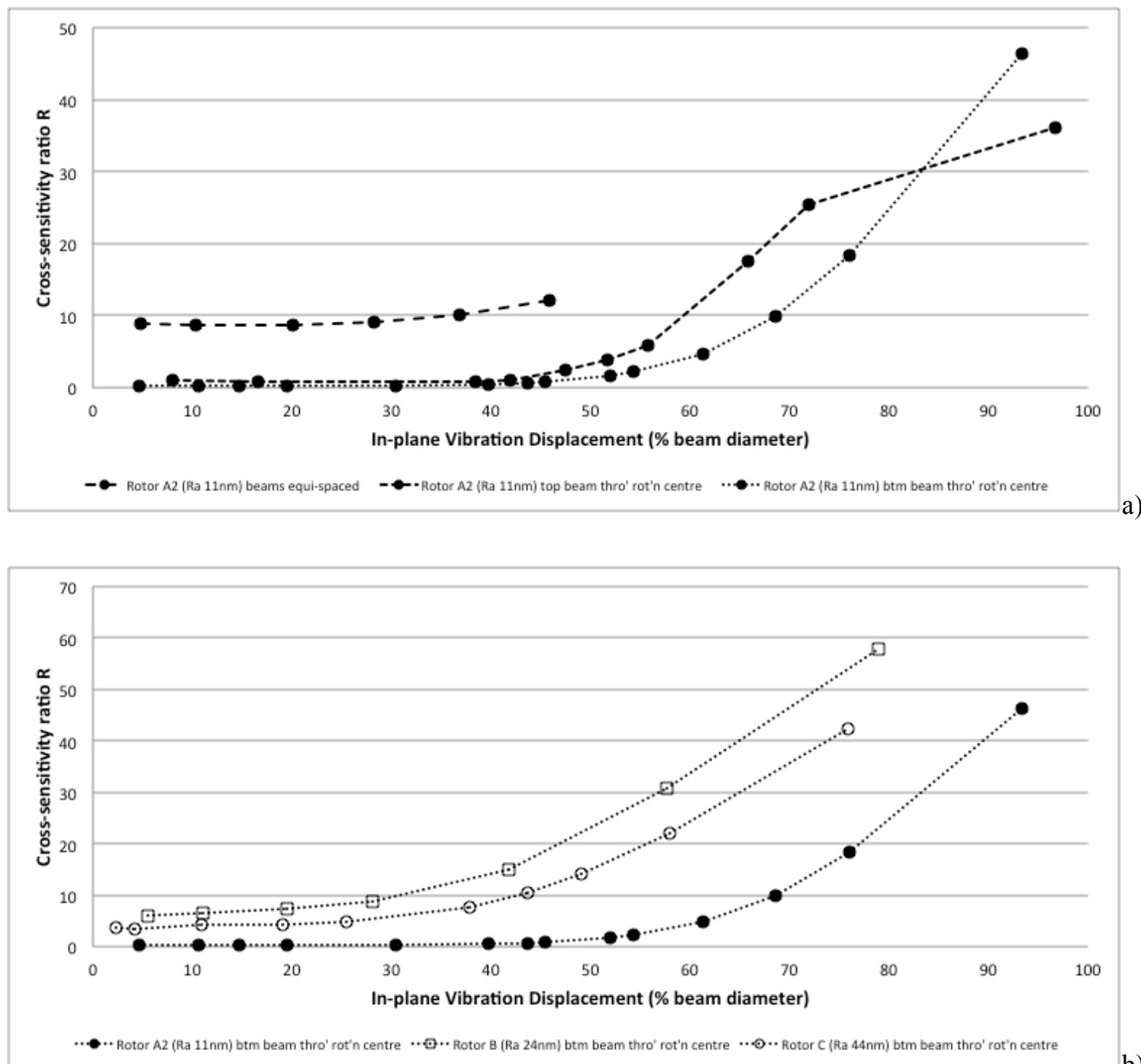

Figure 9. Effect of displacement amplitude ( $520 \mu \mathrm{m}$ beam diameter $-400 \mathrm{~mm}$ stand-off) for rotor A2 (Ra $11 \mathrm{~nm}$ ) for side of rotor measurements; also showing the impact of beam alignment relative to oscillation centre. 


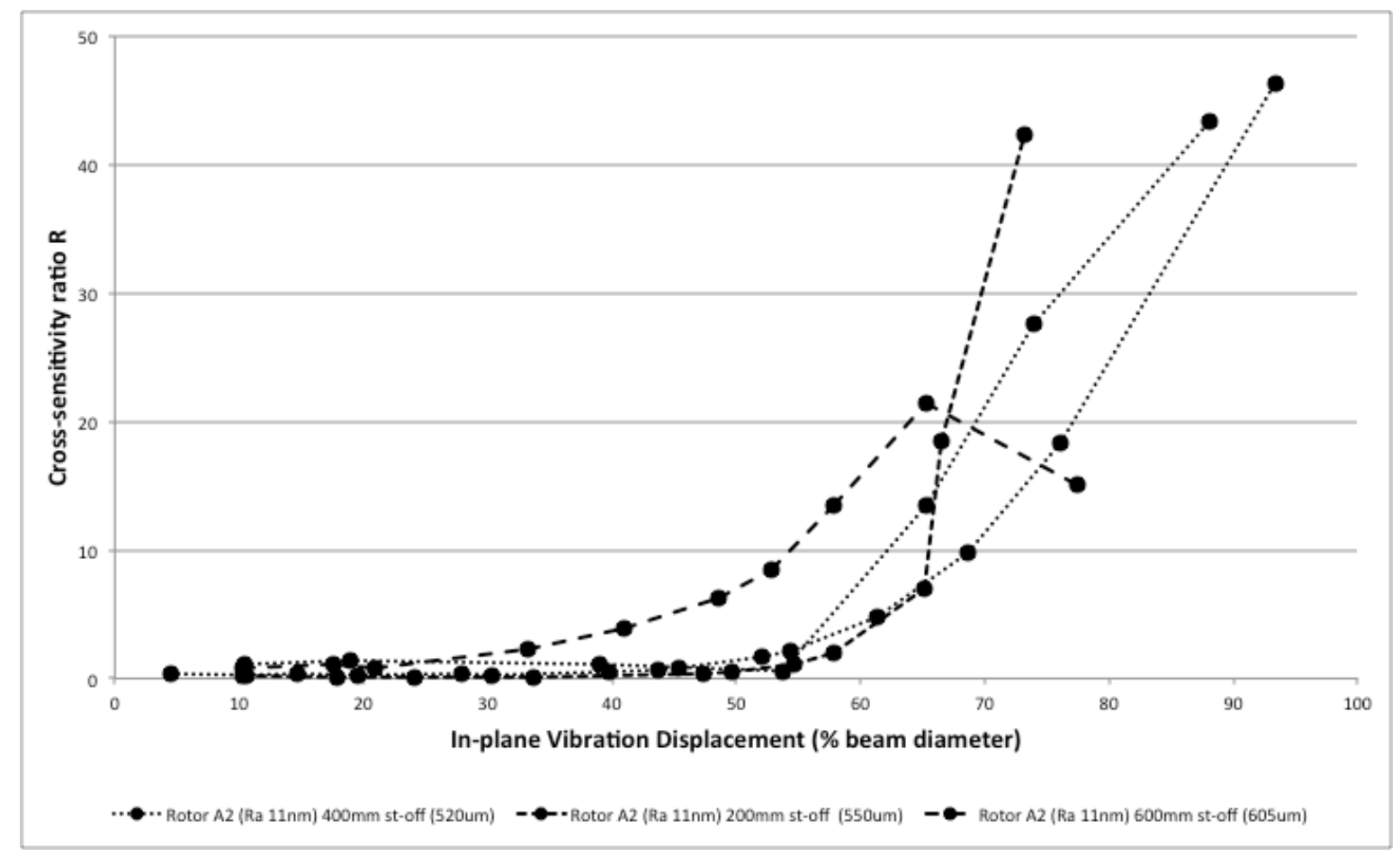

Figure 10. Effect of stand-off distance (and therefore beam diameter) for rotor $\mathrm{A} 2$ (Ra $11 \mathrm{~nm}$ ) for side of rotor measurements. 


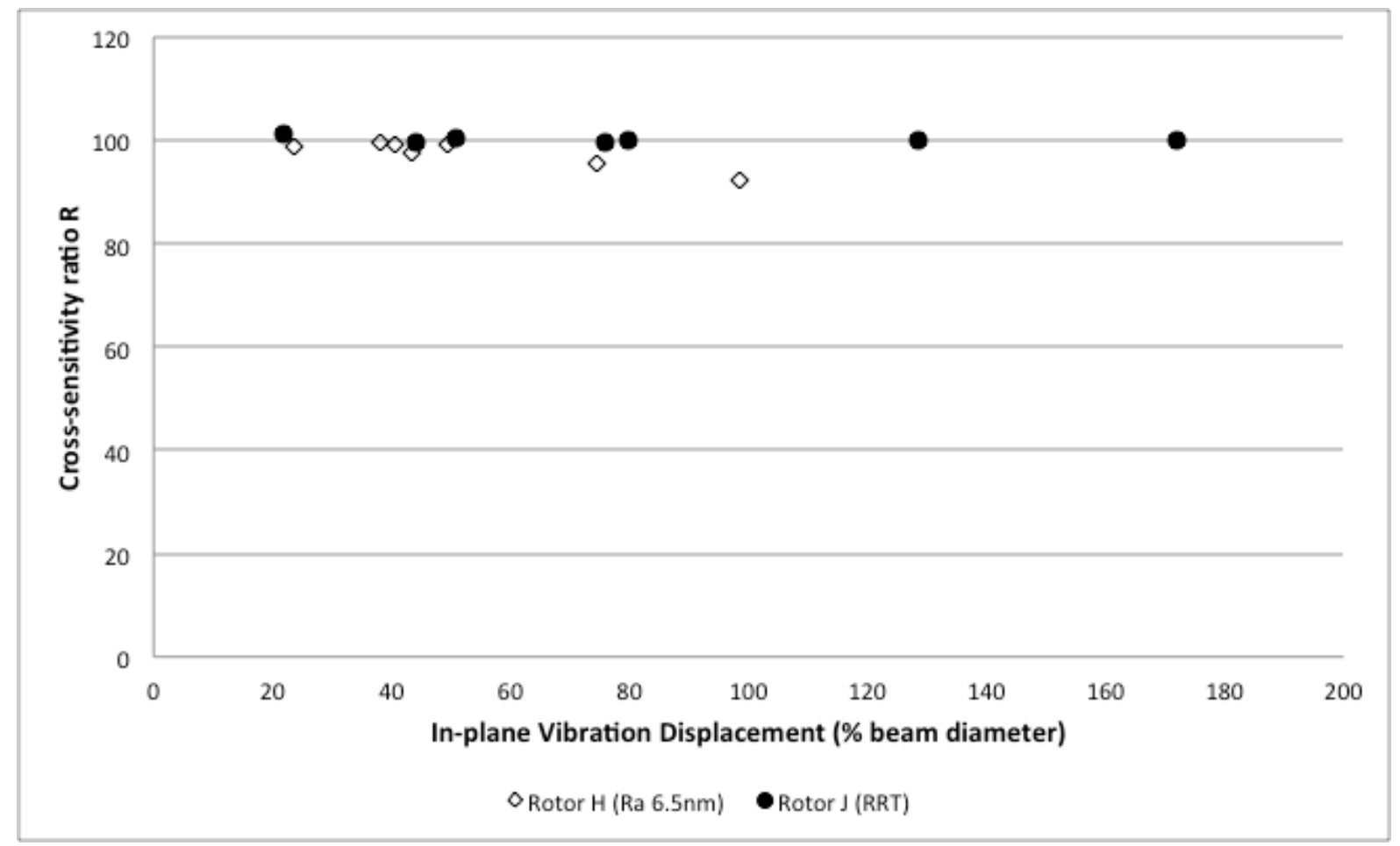

Figure 11. Effect of displacement amplitude (520 $\mu \mathrm{m}$ beam diameter, $400 \mathrm{~mm}$ stand-off) for rotor end face measurements. 


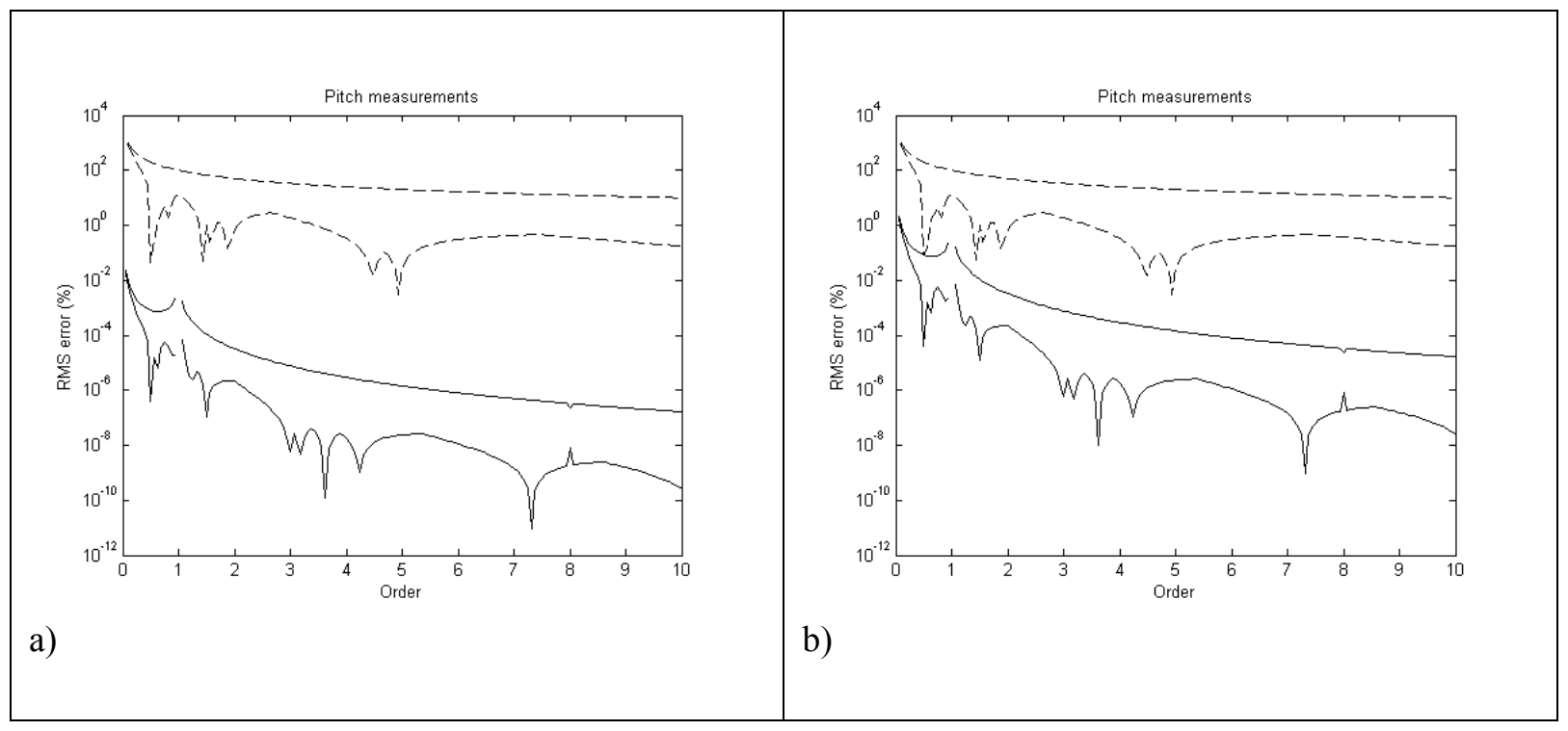

Figure 12. Maximum and minimum errors in measured angular velocity prior to resolution (dashed lines) and after resolution (solid lines) a) $2.5 \mathrm{mrad} \mathrm{s}^{-1} / \mathrm{rad} \mathrm{s}^{-1}$ b) $25 \mathrm{mrad} \mathrm{s}^{-1} / \mathrm{rad} \mathrm{s}^{-1}$. 


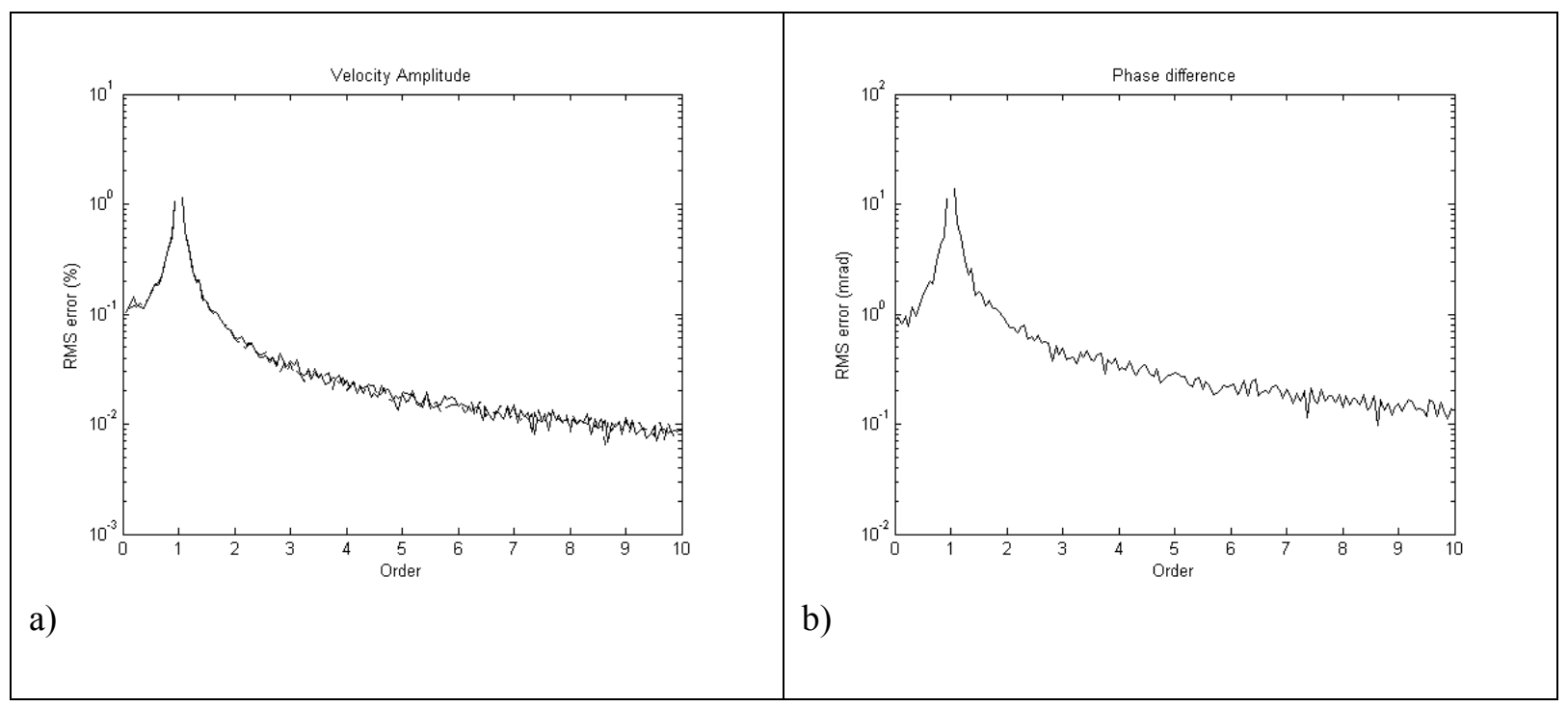

Figure 13. RMS error in resolved angular velocity as a result of broadband speed variation; a) pitch (solid line) and yaw (dashed line) amplitudes and b) phase difference. 


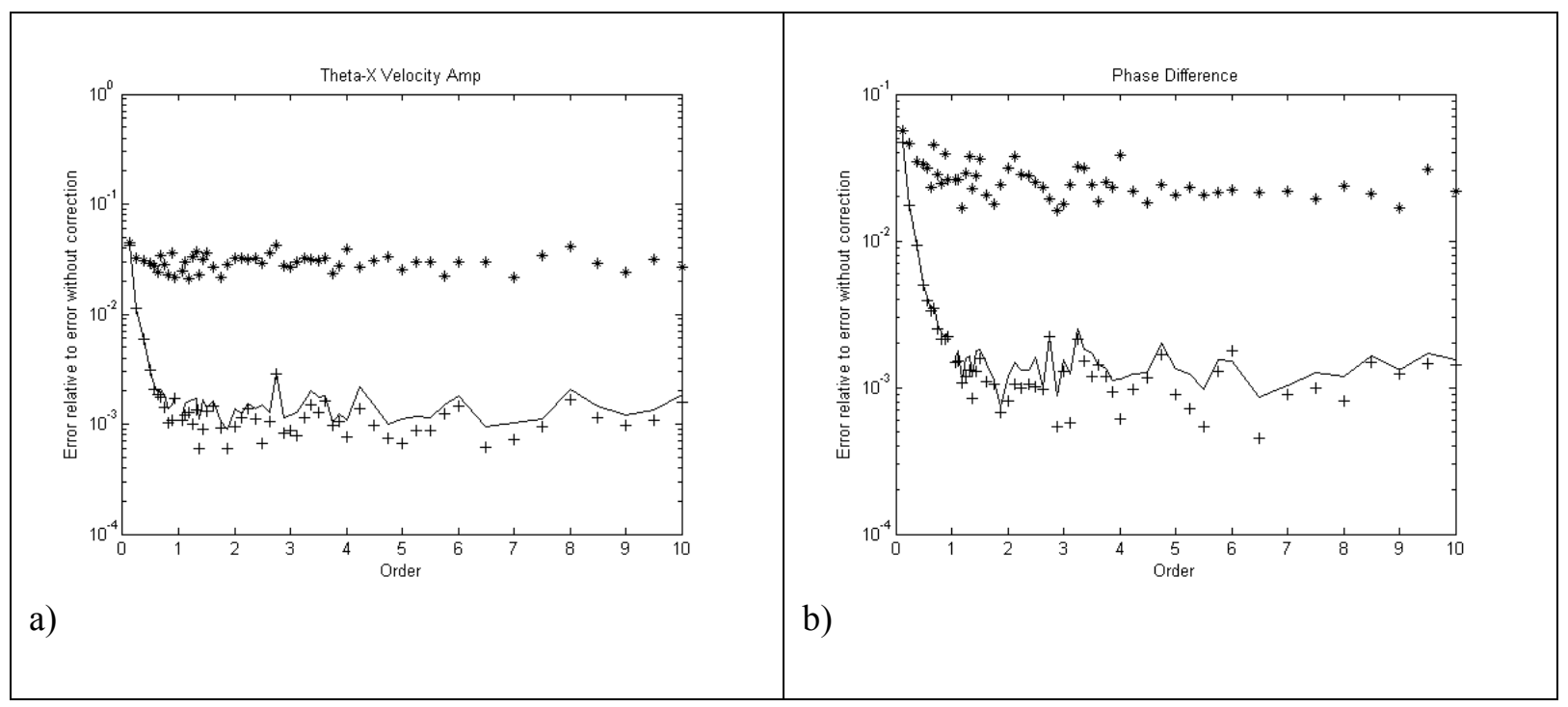

Figure 14. Relative error in resolved velocity following $m$ iterations through the correction algorithm (*) $m=1$, solid line $m=2,(+) m=4$; a) pitch amplitude and b) phase difference. 


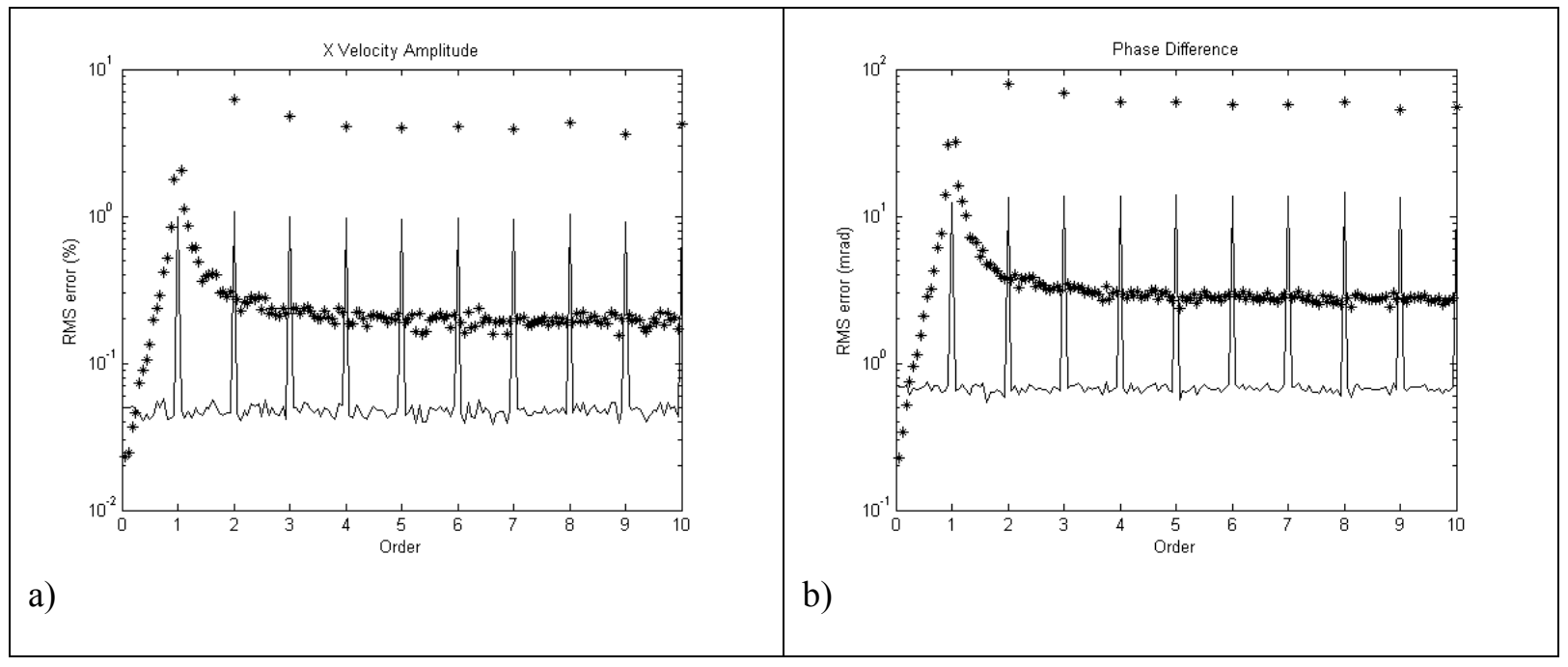

Figure 15. RMS error in resolved angular velocity as a result of pseudo-vibration for polished-circular (solid line) and rough $(*)$ rotor model; a) pitch amplitude and b) phase difference. 


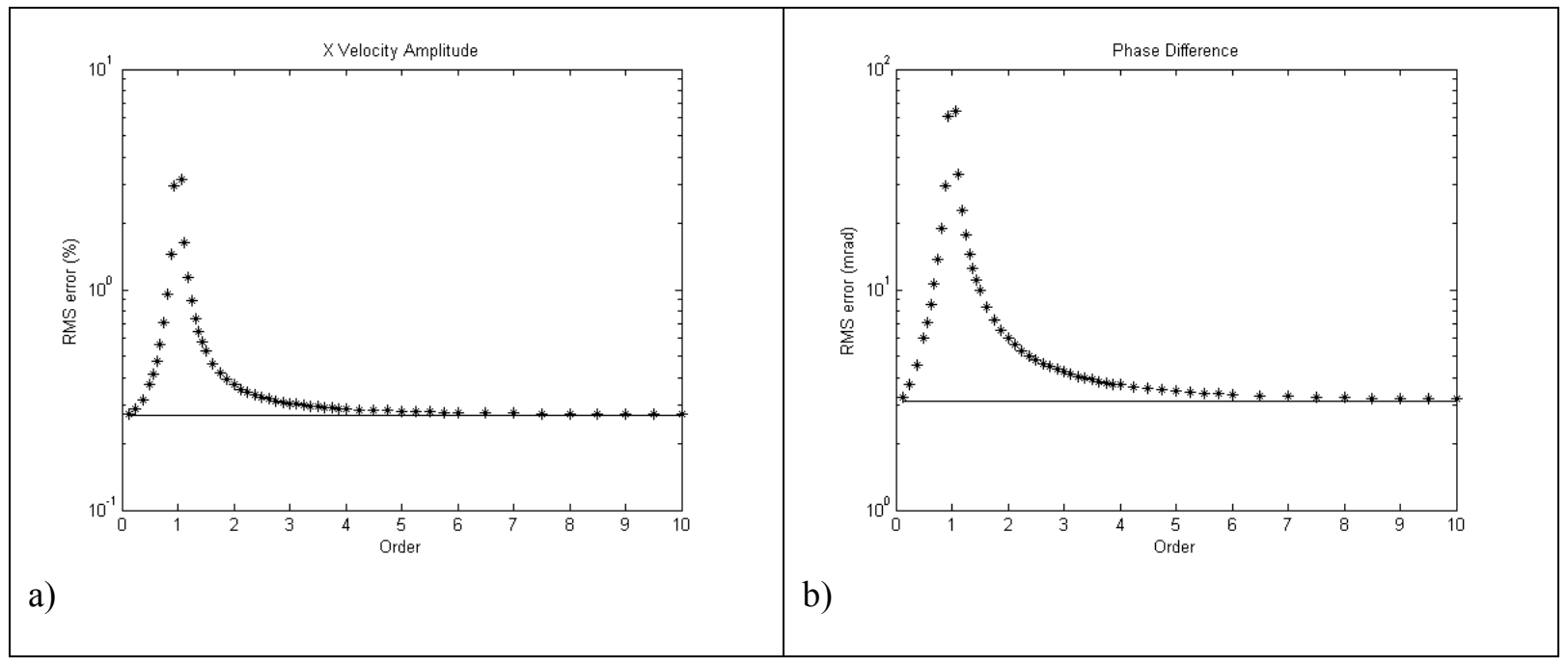

Figure 16. RMS error in resolved angular velocity as a result of misalignments for polished-circular (solid line) and rough $(*)$ rotor model; a) pitch amplitude b) phase difference. 


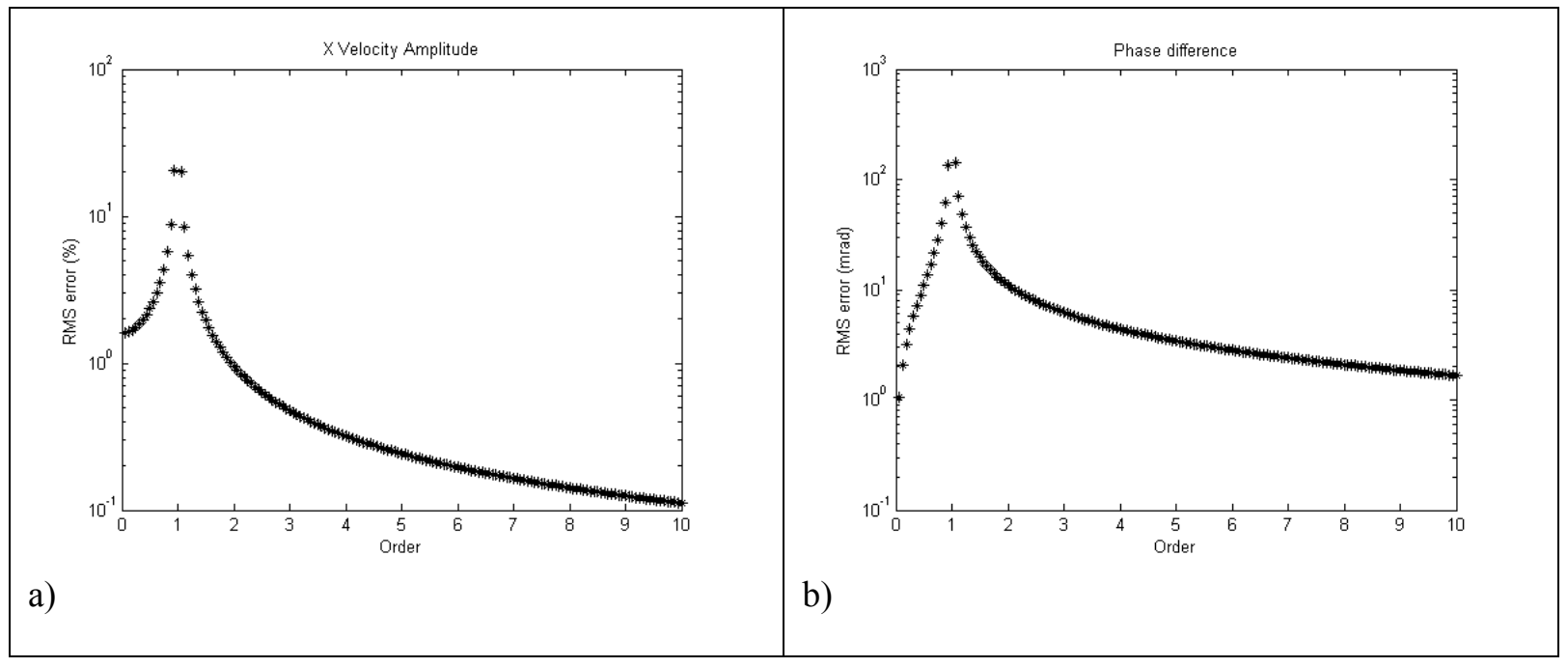

Figure 17. RMS error in resolved angular velocity as a result of speed measurement error for rough rotor model; a) pitch amplitude b) phase difference. 


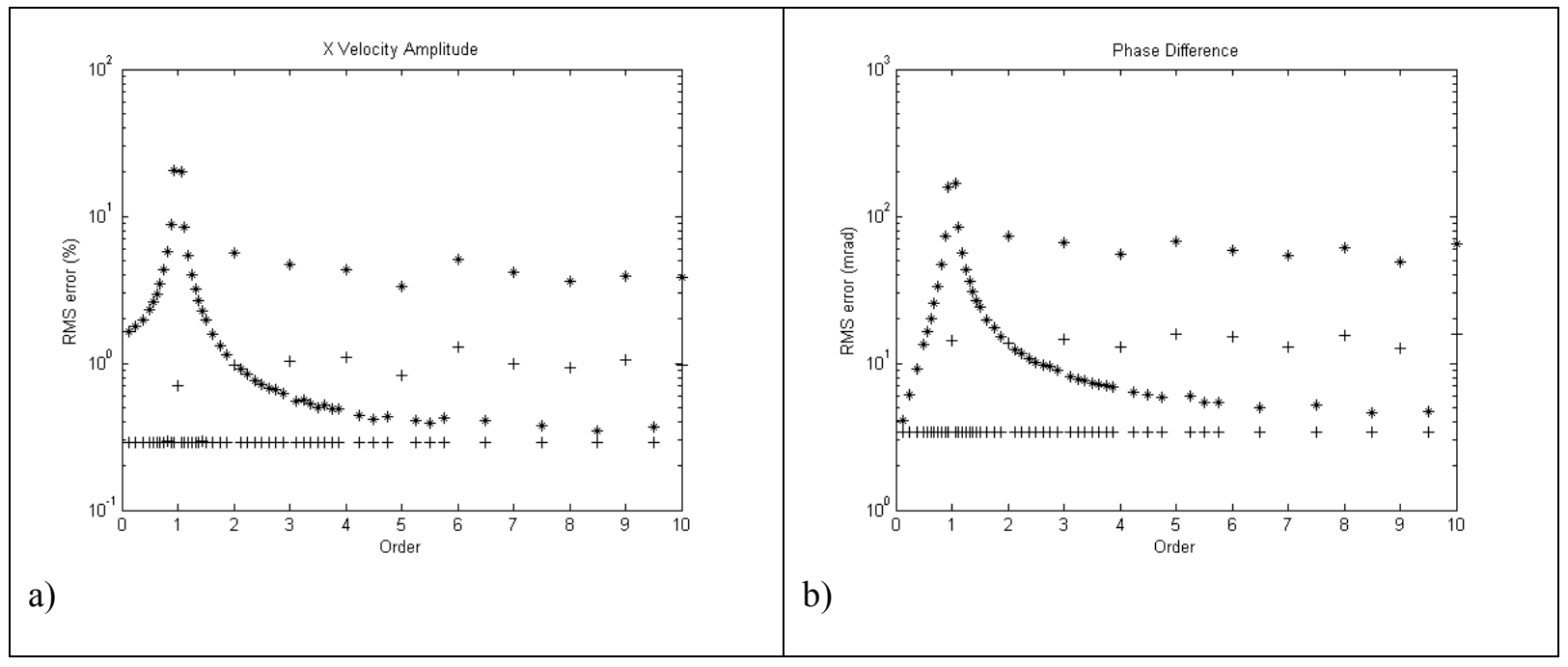

Figure 18. RMS error in resolved angular velocity as a result of the combined effects of pseudovibration, misalignments and speed measurement error for polished-circular $\left(^{*}\right)$ and rough $(+)$ rotor model; a) pitch amplitude b) phase difference. 